\title{
A simple justification of effective models for conducting or fluid media with dilute spherical inclusions
}

\author{
David Gérard-Varet
}

March 16, 2021

\begin{abstract}
We present a gentle approach to the justification of effective media approximations, for PDE's set outside the union of $n \gg 1$ spheres with low volume fraction. To illustrate our approach, we consider three classical examples: the derivation of the so-called strange term, made popular by Cioranescu and Murat, the derivation of the Brinkman term in the Stokes equation, and a scalar analogue of the effective viscosity problem. Under some separation assumption on the spheres, valid for periodic and random distributions of the centers, we recover effective models as $n \rightarrow+\infty$ by simple arguments.
\end{abstract}

\section{Introduction}

Let $B_{i, n}=B\left(x_{i, n}, r_{n}\right), 1 \leq i \leq n$, a collection of disjoint balls, included in a compact subset $K$ of $\mathbb{R}^{3}$. We assume convergence of the empirical measure

$$
\frac{1}{n} \sum_{i=1}^{n} \delta_{x_{i, n}} \rightarrow \rho(x) d x, \quad n \rightarrow+\infty
$$

where $\rho$ is a bounded density with support $K_{\rho} \subset K$. Moreover, we assume that the volume fraction of the balls

$$
\lambda_{n}:=\frac{4 \pi}{3} \frac{n r_{n}^{3}}{\left|K_{\rho}\right|}
$$

is small uniformly in $n$ (and in some cases vanishes as $n$ goes to infinity).

Our concern is the asymptotics of elliptic PDE's of Laplace or Stokes type, in the domain $\Omega_{n}:=\mathbb{R}^{3} \backslash \cup_{i} B_{i, n}:$

$$
L u_{n}=g \quad \text { in } \Omega_{n},
$$

completed with boundary conditions at each ball $B_{i}$ and a decay condition at infinity. Such PDE's arise in various models, taken from various areas: electrostatics, optics, fluid mechanics, heat conduction and many more. Our main three examples will be:

i) Diffusion in domains with holes:

$$
-\Delta u_{n}=g \quad \text { in } \Omega_{n},\left.\quad u_{n}\right|_{B_{i, n}}=0, \quad 1 \leq i \leq n .
$$

ii) Drag in fluid flows around obstacles:

$$
-\Delta u_{n}+\nabla p_{n}=g, \quad \operatorname{div} u_{n}=0 \quad \text { in } \Omega_{n},\left.\quad u_{n}\right|_{B_{i, n}}=0, \quad 1 \leq i \leq n .
$$


iii) Permittivity in composites with perfectly conducting inclusions :

$$
-\Delta u_{n}=g \quad \text { in } \Omega_{n},\left.\quad u_{n}\right|_{B_{i, n}}=u_{i, n}, \quad u_{i, n} \in \mathbb{R}, \quad \int_{\partial B_{i}} \partial_{\nu} u_{n} d \sigma=-\int_{B_{i}} g, \quad 1 \leq i \leq n,
$$

with $\nu$ the unit normal vector pointing outward.

Just like (3) has (4) for fluid counterpart, (5) is the scalar version of the effective viscosity problem

$$
\begin{aligned}
& -\Delta u_{n}+\nabla p_{n}=g, \quad \operatorname{div} u_{n}=0 \quad \text { in } \Omega_{n},\left.\quad u_{n}\right|_{B_{i, n}}=u_{i, n}+\omega_{i, n} \times\left(x-x_{i, n}\right) \quad u_{i, n}, \omega_{i, n} \in \mathbb{R}^{3}, \\
& \int_{\partial B_{i}}\left(2 D\left(u_{n}\right) \nu-p \nu\right) d \sigma=-\int_{B_{i}} g, \\
& \int_{\partial B_{i}}\left(2 D\left(u_{n}\right) \nu-p \nu\right)(x) \times\left(x-x_{i}\right) d \sigma(x)=-\int_{B_{i}} g(x) \times\left(x-x_{i}\right) d x, \quad 1 \leq i \leq n,
\end{aligned}
$$

that could be included in our analysis as well. Other problems fit a similar framework, such as the propagation of waves in bubbly fluids [3].

Back to the general formulation (2), the point is to determine if, for large $n$ and small volume fraction, the solution $u_{n}$ is close to the solution $u$ of an effective model:

$$
L u+L_{e} u=0 \quad \text { in } \mathbb{R}^{3}
$$

where the extra effective operator $L_{e}$ reflects some average effect of the spheres. Moreover, the hope is to express $L_{e}$ in terms of macroscopic characteristics of the spheres distribution, like the limit density $\rho$ and the limit volume fraction $\lambda:=\lim _{n} \lambda_{n}$ (when non zero). This hope is supported by the following formal reasoning. If the volume fraction of spherical inclusions is small, one can expect the average distance between the spheres to be large compared to their radius. Therefore, the interaction of the spheres should be negligible, and their contribution to the effective operator should be additive. Furthermore, if the volume fraction vanishes as $n$ grows, the spheres should be well approximated by points. By combining both arguments, the leading effect of the spheres at large $n$ should be through the empirical measure. In the limit $n \rightarrow+\infty$, one should then recover $L_{e}=L_{e}[\rho]$.

In this spirit, early formal calculations of effective models were done by Maxwell Garnett [29], Clausius [9], Mossotti [31], to describe heterogeneous electromagnetic media. Similar computation of the effective viscosity of dilute suspensions was done by Einstein [16]. For the drag generated by obstacles in a fluid flow, see Brinkman [5]. Transposed to our three examples, these calculations lead to the following lose statements:

i) For system (3), the critical scaling is $r_{n}=\frac{1}{n}$, with convergence of $u_{n}$ to the solution $u$ of

$$
-\Delta u+4 \pi \rho u=g \quad \text { in } \mathbb{R}^{3} .
$$

ii) For system (4), the critical scaling is $r_{n}=\frac{1}{n}$, with convergence of $u_{n}$ to the solution $u$ of

$$
-\Delta u+\nabla p+6 \pi \rho u=g, \quad \operatorname{div} u=0 \text { in } \mathbb{R}^{3} .
$$

iii) For system (5), the critical scaling is when $\lambda:=\lim _{n} \lambda_{n}$ is small but non zero. One has in this case $u_{n}=u+o(\lambda)$ uniformly in $n$, with

$$
-\operatorname{div}\left(\left(1+3 \lambda\left|K_{\rho}\right| \rho\right) \nabla u\right)=g \quad \text { in } \mathbb{R}^{3} .
$$


By critical scaling, we mean that other scalings would lead to trivial limits: typically, in system (3), one has $u=0$ if $r_{n} \gg \frac{1}{n}$, while it solves the original Laplace equation if $r_{n} \ll \frac{1}{n}$.

Turning these assertions into rigorous mathematical statements has attracted a lot of attention since 1970. Pioneering results on (3) are due to Hruslov and Marchenko [28], Cioranescu and Murat [8], Papanicolaou and Varadhan [34], or Ozawa [33]. A more abstract approach through $\Gamma$-convergence was completed in $[10,11]$. For the most up to date statements, we refer to the nice study by Giunti, Höfer and Velázquez [20], and to the bibliography therein. On the asymptotics of (4), one can mention the work of Allaire [1], as well as improvements by Desvillettes, Golse and Ricci[14], Hillairet [23], or Höfer [25]. As regards the analysis of (5) and of the more involved fluid problem (6), rigorous results were obtained in Levy and Sanchez-Palencia [35, 27], Ammari et al [2], Haines and Mazzucato [22], as well as in the recent papers by Hillairet and $\mathrm{Wu}$ [24], Niethammer and Schubert [32] , or the author and Hillairet [18]. These references are by no mean exhaustive: one could further cite $[26,6,7,30,15,21]$ and many more on related problems.

As explained before, the derivation of the limit systems (8), (9) and (10) is based on neglecting the interaction between the spheres. It requires some separation assumption on the centers $x_{i, n}$ of the spheres. The challenge is to show convergence under the mildest possible separation assumption. This is what differentiates the works mentioned above. Three types of assumptions emerge from the literature:

- the most stringent one is that the centers are periodically distributed over a mesh of typical length $n^{-1 / 3}$. This is assumed for instance in [8], [1] or in the studies [35, 27] as well as in [22]. Note that in such a case, the limit density $\rho$ is constant over its support: $\rho=\left|K_{\rho}\right|^{-1} 1_{K_{\rho}}$.

- Other studies relax the periodicity assumption, but keep in addition to (H0) a condition on the minimal distance between the centers:

$$
\inf _{i \neq j}\left|x_{i, n}-x_{j, n}\right| \geq c n^{-1 / 3} .
$$

This is the case in [33], or in [14], and as far as we know in all works on the effective permittivity problem (5) or on the effective viscosity problem (6). Although it is an improvement to the periodic case, such assumption is not fully satisfactory: in particular, it is not satisfied if the centers are drawn randomly and independently with law $\rho(x) d x$. Indeed, in this latter case, it is known that the typical minimal distance scales like $n^{-2 / 3}$.

- Eventually, the asymptotics of system (3) or (4) may be established under weaker assumptions, which apply when the centers $x_{i, n}$ are given by i.i.d. random variables, or derived from reasonable stationary point processes. A big step in that direction was made in [34]: roughly ${ }^{1}$, the solution $u_{n}$ of (3) tends to the solution $u$ of (8) under the weak separation assumption

$$
\frac{1}{n^{2}} \sum_{i \neq j} \frac{1}{\left|x_{i, n}-x_{j, n}\right|} \rightarrow \int_{\mathbb{R}^{3} \times \mathbb{R}^{3}} \frac{1}{|x-y|} \rho(x) \rho(y) d x d y, \quad n \rightarrow+\infty
$$

It is shown in [34] that this convergence holds in probability in the i.i.d. case. Recently, almost sure convergence of $u_{n}$ to $u$ was proved in [20] for a large class of stationary point processes, allowing for random radii of the spheres. See also [19] for the analogue in the Stokes case.

\footnotetext{
${ }^{1}$ actually, [34] treats the time dependent version of the equations
} 
The works mentioned above may rely on very different techniques. Still, key arguments are often difficult and based on explicit constructions. For instance, the work of Cioranescu and Murat, as well as the refined analyses [1,20], are based on the construction of so-called correctors, which may be quite technical notably in the Stokes case. Another instance is [34], where the proof relies on the explicit representation of $u_{n}$ with the Feynman-Kac formula, and on non-trivial manipulations of this formula. In particular, none of these methods really exploits the fact that the formal limit equation is already known. The goal of this paper is to present a softer and shorter approach to these asymptotic problems. This approach relies on separation hypotheses in the same spirit as (12), although slightly stronger. In the context of problem (3), resp. (4), our assumptions (H1)-(H2), resp. (H1)-(H2'), see next section, cover the periodic and random i.i.d. cases. In the context of (5), we derive the effective permittivity formula under a couple of conditions (A1)-(A2) that is more general than (11), and to our knowledge is new. Still, we insist that the point of this paper is not the novelty of our statements, for which refined versions often exist. Our goal is rather to give a less constructive method than in former works, which results in much shorter proofs.

\section{Strategy and hypotheses for the convergence}

Let us explain our idea to go rigorously from (2) to (7). Given a smooth and compactly supported test field $\varphi$ on $\mathbb{R}^{3}$, the point is to introduce a solution $\phi_{n}=\phi_{n}[\varphi]$ of

$$
L^{*} \phi_{n}=-L_{e}^{*} \varphi \quad \text { in } \Omega_{n}
$$

completed with appropriate boundary conditions at $\partial \Omega_{n}$. By appropriate, we mean that for natural extensions of $u_{n}$ and $\phi_{n}$ inside $\cup_{i} B_{i, n}$, still denoted $u_{n}$ and $\phi_{n}$, one has the identity

$$
\int_{\Omega_{n}}\left(L u_{n}-g\right)\left(\varphi-\phi_{n}\right)=\int_{\mathbb{R}^{3}}\left(L u_{n}+L_{e} u_{n}-g\right) \varphi+\int_{\mathbb{R}^{3}} g \phi_{n}
$$

which in the case without boundaries would come from the formal calculations:

$$
\int L u_{n}\left(\varphi-\phi_{n}\right)=\int L u_{n} \varphi-\int u_{n} L^{*} \phi_{n}=\int L u_{n} \varphi+\int u_{n} L_{e}^{*} \varphi=\int\left(L+L_{e}\right) u_{n} \varphi .
$$

By (2), it will result from (14) that

$$
\int_{\mathbb{R}^{3}}\left(L u_{n}+L_{e} u_{n}-g\right) \varphi+\int_{\mathbb{R}^{3}} g \phi_{n}=0 .
$$

Hence, any accumulation point $u$ of $u_{n}$ satisfies

$$
\left|\int_{\mathbb{R}^{3}}\left(L u+L_{e} u-g\right) \varphi\right| \leq \underset{n}{\limsup }\left|\int_{\mathbb{R}^{3}} g \phi_{n}\right|
$$

To obtain (7) in the limit $n \rightarrow+\infty$, or up to $o(\lambda)$ term, it is then enough to show that $\phi_{n}$ converges to 0 weakly, or up to a $o(\lambda)$ term.

Let us remark that in the case of systems (3) and (4), our solution $\phi_{n}$ is connected to the abstract test function $w^{\varepsilon}$ that appears in [8] for the derivation of the strange term, or in [1] for the derivation of the Brinkman term. We shall expand on this connection in Remark 4 below. Nevertheless, in both [8] and [1] , the concrete construction of $w^{\varepsilon}$ is quite tedious (and restricted to the periodic framework). Here, the direct introduction of $\phi_{n}$ simplifies the derivation: its convergence to zero relies on relatively soft arguments, in contrast with the use of correctors. 
Namely, in the case where $L_{e}=L_{e}[\rho]$, the idea is that

$$
\phi_{n} \approx \phi_{n}^{1}, \quad \phi_{n}^{1}:=G^{*} \star\left(-L_{e}^{*}[\rho] \varphi\right)-G^{*} \star\left(-L_{e}^{*}\left[n^{-1} \sum \delta_{x_{i}}\right] \varphi\right)
$$

with $G^{*}$ the fundamental solution of $L^{*}$. The first term in the definition of $\phi_{n}^{1}$ is to take care of the source term in (13), while the second one is to take care of the boundary conditions at the spheres, neglecting the interaction between them. Note that $\phi_{n}^{1}$ goes formally to zero under the simple assumption (H0). Actually, the main point, from which separation assumptions stem, is to show that $\phi_{n}-\phi_{n}^{1}$ vanishes as $n \rightarrow+\infty$, or is $o(\lambda)$. Assumptions on the separation between the centers stem from this requirement.

More precisely, in the context of system (3), with $r_{n}=\frac{1}{n}$, our assumptions are

$$
\exists c>2, \quad \forall i \neq j, \quad\left|x_{i}-x_{j}\right| \geq \frac{c}{n},
$$

$\limsup _{n \rightarrow+\infty} \sum_{i} \int_{B_{i}}\left(n^{2}\left|\frac{1}{n} \sum_{j \neq i} G\left(x-x_{j}\right)-\int_{\mathbb{R}^{3}} G(x-y) \rho(y) d y\right|^{2}+\frac{1}{n^{2}}\left|\sum_{j \neq i} \nabla G\left(x-x_{j}\right)\right|^{2}\right) d x=0$.

We have noted $x_{i}:=x_{i, n}, B_{i}:=B_{i, n}$ for short, and $G(x):=\frac{1}{4 \pi|x|}$ the kernel of $-\Delta$. Settings in which (H1)-(H2) are satisfied, including periodic and random i.i.d. cases, will be discussed in the last section 4. Let us already point out that (H1) and (H2) both express some kind of separation on the centers $x_{i, n}$. While (H1) is a mild requirement on their minimal distance, (H2) is a condition of non-clustering in the mean, in the spirit of (12). Let us note that if $G$ were smooth, (H2) would be a simple consequence of (H0). The point here is the singularity of $G$ at the origin, which penalizes points $x_{i}, x_{j}$ close from one another. We state

Theorem 1. Let $g \in L^{\frac{6}{5}}\left(\mathbb{R}^{3}\right), r_{n}=\frac{1}{n}$. Under (H0)-(H1)-(H2), the solution $u_{n}$ of $(3)$ converges weakly in $\dot{H}^{1}\left(\mathbb{R}^{3}\right) \cap L^{6}\left(\mathbb{R}^{3}\right)$ to the solution $u$ of $(8)$.

In the context of the Stokes equation (4), assumption (H2) must be modified into

$$
\begin{gathered}
\limsup _{n \rightarrow+\infty} \sum_{i} \int_{B_{i}}\left(n^{2}\left|\frac{1}{n} \sum_{j \neq i} G_{S t}\left(x-x_{j}\right)-\int_{\mathbb{R}^{3}} G_{S t}(x-y) \rho(y) d y\right|^{2}+\frac{1}{n^{2}}\left|\sum_{j \neq i} \nabla G_{S t}\left(x-x_{j}\right)\right|^{2}\right. \\
\left.+\frac{1}{n^{4}}\left|\sum_{j \neq i} R_{S t}\left(x-x_{j}\right)\right|^{2}+\frac{1}{n^{6}}\left|\sum_{j \neq i} \nabla R_{S t}\left(x-x_{j}\right)\right|^{2}\right) d x=0
\end{gathered}
$$

with $G_{S t}(x)=\frac{1}{8 \pi}\left(\frac{I}{|x|}+\frac{x \otimes x}{|x|^{3}}\right)$ the kernel of $-\mathbb{P} \Delta$, and $R_{s t}(x)=\frac{1}{8 \pi}\left(\frac{I}{3|x|^{3}}-\frac{x \otimes x}{|x|^{5}}\right)$.

Theorem 2. Let $g \in L^{\frac{6}{5}}\left(\mathbb{R}^{3}\right)^{3}, r_{n}=\frac{1}{n}$. Under (H0)-(H1)-(H2'), the solution $u_{n}$ of (4) converges weakly in $\dot{H}^{1}\left(\mathbb{R}^{3}\right)^{3} \cap L^{6}\left(\mathbb{R}^{3}\right)^{3}$ to the solution $u$ of $(9)$.

Eventually, system (5) can be analyzed in the regime where the volume fraction $\lambda_{n}=\lambda$ is small but independent of $n$. We rely this time on assumptions

$$
\exists c>2, \quad \forall i \neq j, \quad\left|x_{i}-x_{j}\right| \geq c r_{n},
$$

and for all smooth $\varphi$,

$$
\limsup _{n \rightarrow+\infty} \frac{1}{n^{2}} \sum_{i} \int_{B_{i}}\left|\sum_{j \neq i} \nabla V\left(x-x_{j}\right) \nabla \varphi\left(x_{j}\right)\right|^{2} d x \leq \eta(\lambda)\|\nabla \varphi\|_{L^{\infty}}, \quad \eta(\lambda) \underset{\lambda \rightarrow 0}{\longrightarrow} 0,
$$

where $V(x)=\nabla \frac{1}{4 \pi|x|}$. This last assumption will be discussed further in Section 4 . It will be notably shown to be implied by the usual condition (11). We shall prove 
Theorem 3. Let $g \in L^{\frac{6}{5}}\left(\mathbb{R}^{3}\right)$. Assume $\lambda_{n}=\lambda$ for all $n$. Let $u_{n, \lambda}$ the solution of (5). Under (H0)-(A1)-(A2), any accumulation point $u_{\lambda}$ of $u_{n, \lambda}$ in $\dot{H}^{1}\left(\mathbb{R}^{3}\right) \cap L^{6}\left(\mathbb{R}^{3}\right)$ solves

$$
-\operatorname{div}\left(\left(1+3 \lambda\left|K_{\rho}\right| \rho\right) \nabla u\right)=g+R_{\lambda} \quad \text { in } \mathbb{R}^{3},
$$

with a remainder $R_{\lambda}$ satisfying

$$
\left\langle R_{\lambda}, \varphi\right\rangle \leq o(\lambda)\|\nabla \varphi\|_{L^{\infty}}, \quad \forall \varphi \in \dot{H}^{1}\left(\mathbb{R}^{3}\right) \cap L^{6}\left(\mathbb{R}^{3}\right) \cap \operatorname{Lip}\left(\mathbb{R}^{3}\right) .
$$

\section{Proofs}

\subsection{Proof of Theorem 1}

We extend $u_{n}$ by zero inside the balls, and still denote $u_{n}$ this extension. A simple energy estimate of (3) together with Sobolev imbedding yields

$$
\left\|\nabla u_{n}\right\|_{L^{2}\left(\mathbb{R}^{3}\right)}^{2} \leq\|g\|_{L^{\frac{6}{5}\left(\mathbb{R}^{3}\right)}}\left\|u_{n}\right\|_{L^{6}\left(\mathbb{R}^{3}\right)} \leq C\|g\|_{L^{\frac{6}{5}\left(\mathbb{R}^{3}\right)}}\left\|\nabla u_{n}\right\|_{L^{2}\left(\mathbb{R}^{3}\right)}
$$

so that $u_{n}$ is bounded in $\dot{H}^{1}\left(\mathbb{R}^{3}\right) \cap L^{6}\left(\mathbb{R}^{3}\right)$. Up to a subsequence in $n$, it has a weak limit $u$. Now, given $\varphi \in C_{c}^{\infty}\left(\mathbb{R}^{3}\right)$, we introduce $\phi_{n}$ the solution of

$$
-\Delta \phi_{n}=-4 \pi \rho \varphi \quad \text { in } \Omega_{n},\left.\quad \phi_{n}\right|_{\cup_{i} B_{i}}=\varphi .
$$

Taking $\varphi-\phi_{n}$ as a test function in (3), we get by Green's formula:

$$
\int_{\mathbb{R}^{3}} \nabla u_{n} \cdot \nabla \varphi+4 \pi \int_{\mathbb{R}^{3}} \rho u_{n} \varphi=\int_{\mathbb{R}^{3}} g \varphi-\int_{\mathbb{R}^{3}} g \phi_{n} .
$$

We have used here that $u_{n}$ and $\varphi-\phi_{n}$ vanish inside the balls to replace integrals over $\Omega_{n}$ by integrals over $\mathbb{R}^{3}$. Now, if we prove that the last term at the right-hand side goes to zero, then $u \in \dot{H}^{1}\left(\mathbb{R}^{3}\right) \cap L^{6}\left(\mathbb{R}^{3}\right)$ will be a variational solution of (8). As this solution is unique, the full sequence $u_{n}$ will converge to $u$, proving the theorem. As $g$ is arbitrary in $L^{\frac{6}{5}}\left(\mathbb{R}^{3}\right)$, we need to prove that $\phi_{n}$ converges weakly to zero in $L^{6}\left(\mathbb{R}^{3}\right)$. By a standard energy estimate on $\phi_{n}-\varphi$, which vanishes at the balls, it is easily seen that $\phi_{n}$ is bounded in $\dot{H}^{1}\left(\mathbb{R}^{3}\right) \cap L^{6}\left(\mathbb{R}^{3}\right)$, so that it is enough to show convergence of $\phi_{n}$ to zero in the sense of distributions.

Actually, we can further simplify the analysis by introducing the solution $\Phi_{n}$ of

$$
-\Delta \Phi_{n}=-4 \pi \rho \quad \text { in } \Omega_{n},\left.\quad \Phi_{n}\right|_{\cup_{i} B_{i}}=1 .
$$

Indeed, let $R_{n}=\phi_{n}-\Phi_{n} \varphi$, that satisfies

$$
-\Delta R_{n}=2 \nabla \Phi_{n} \cdot \nabla \varphi+\Phi_{n} \Delta \varphi \quad \text { in } \Omega_{n},\left.\quad R_{n}\right|_{\cup_{i} B_{i}}=0 .
$$

If we prove that $\Phi_{n} \rightarrow 0$ strongly in $L_{l o c}^{2}$, then, by a standard energy estimate, $R_{n} \rightarrow 0$ strongly in $H_{l o c}^{1}$, and eventually $\phi_{n} \rightarrow 0$ strongly in $L_{l o c}^{2}$.

For $\eta>0$, we denote $\delta_{n}^{\eta}=\frac{1}{4 \pi \eta^{2} n} \sum_{i} s_{\eta}\left(\cdot-x_{i}\right)$ where $s_{\eta}$ is the surface measure on the sphere of radius $\eta$. To prove strong convergence of $\Phi_{n}$ to zero in $L_{l o c}^{2}$, we split $\Phi_{n}=\Phi_{n}^{1}+\Phi_{n}^{2}$, where

$$
\Phi_{n}^{1}=4 \pi \sum_{i} \mathcal{G}\left(n\left(x-x_{i}\right)\right)-4 \pi G \star \rho
$$

with $G(x)=\frac{1}{4 \pi|x|}$ the fundamental solution of $-\Delta, \mathcal{G}(x)=G(x)$ if $|x| \geq 1, \mathcal{G}(x)=\frac{1}{4 \pi}$ if $|x| \leq 1$. We compute

$$
-\Delta \Phi_{n}^{1}=4 \pi \delta_{n}^{1 / n}-4 \pi \rho \quad \text { in } \mathbb{R}^{3}
$$


and $\Phi_{n}^{1}(x)=1+\frac{4 \pi}{n} \sum_{j \neq i} G\left(x-x_{j}\right)-4 \pi \int_{\mathbb{R}^{3}} G(x-y) \rho(y) d y$ for $x \in B_{i}$, for all $i$. Hence,

$$
-\Delta \Phi_{n}^{2}=0 \quad \text { in } \Omega_{n},\left.\quad \Phi_{n}^{2}\right|_{B_{i}}(x)=-\frac{4 \pi}{n} \sum_{j \neq i} G\left(x-x_{j}\right)+4 \pi \int_{\mathbb{R}^{3}} G(x-y) \rho(y) d y
$$

Now, we use that under assumption (H1), there is $C>0$ such that for all $w=\left(w_{i}\right)_{1 \leq i \leq n}$ in $\prod_{i} H^{1}\left(B_{i}\right)$, the solution $u[w]$ of

$$
-\Delta u[w]=0 \quad \text { in } \Omega_{n},\left.\quad u[w]\right|_{B_{i}}=w_{i} \text { for all } i
$$

satisfies

$$
\|\nabla u[w]\|_{L^{2}\left(\mathbb{R}^{3}\right)}^{2} \leq C \sum_{i}\left(n^{2}\left\|w_{i}\right\|_{L^{2}\left(B_{i}\right)}^{2}+\left\|\nabla w_{i}\right\|_{L^{2}\left(B_{i}\right)}^{2}\right)
$$

We refer to Lemma 6 for a proof in the slightly more difficult Stokes case. Note that the factor $n^{2}$ at the right-hand side of the first inequality is consistent with scaling considerations. By applying this inequality with $w_{i}=-\frac{4 \pi}{n} \sum_{j \neq i} G\left(x-x_{j}\right)+4 \pi \int_{\mathbb{R}^{3}} G(x-y) \rho(y) d y$, noticing that $\sum_{i} \int_{B_{i}}\left|\int_{\mathbb{R}^{3}} \nabla G(x-y) \rho(y) d y\right|^{2} d x=O\left(\frac{1}{n^{2}}\right)$, and combining with (H2), we find that $\left\|\nabla \Phi_{n}^{2}\right\|_{L^{2}\left(\mathbb{R}^{3}\right)}$ goes to zero, so that $\Phi_{n}^{2}$ goes to zero strongly in $H_{l o c}^{1}$.

The last step is to show that $\Phi_{n}^{1}$ goes strongly to zero in $L_{l o c}^{2}$. As the right-hand side of $(20)$ is bounded in $W^{-1, p}\left(\mathbb{R}^{3}\right)$ for any $p<\frac{3}{2}, \Phi_{n}^{1}$ is bounded in $W_{l o c}^{1, p}$ for any $p<\frac{3}{2}$, with compact embedding in $L_{l o c}^{q}$ for any $q<3$. Hence, it is enough to prove convergence to zero in the sense of distributions. Let $\psi \in C_{c}^{\infty}\left(\mathbb{R}^{3}\right)$. Clearly the function $\Psi=\Delta^{-1} \psi$ is well-defined and smooth. We get

$$
\int_{\mathbb{R}^{3}} \Phi_{n}^{1} \psi=\int_{\mathbb{R}^{3}} \Phi_{n}^{1} \Delta \Psi=\left\langle\Delta \Phi_{n}^{1}, \Psi\right\rangle=4 \pi\left\langle\rho-\delta_{n}^{1 / n}, \Psi\right\rangle \rightarrow 0
$$

by the assumption (H0). This concludes the proof.

Remark 4. Readers familiar with article [8] may have recognized a connection between the solution $\Phi_{n}$ of (18), and the abstract couple $\left(w^{\varepsilon}, \mu\right)$ involved in assumptions (H.1)-(H.5) of [8]. Namely, setting $\varepsilon=r_{n}$, one can check that $\Phi_{n}=1-w^{\varepsilon}$ and $\mu=4 \pi \rho$. However, the approach in the present paper remains quite distinct from the one in [8]. In [8], the construction of the corrector $w^{\varepsilon}$ is quite technical, and the identification of $\mu$ is done a posteriori, once $w^{\varepsilon}$ has been built. Here, $\Phi_{n}$ (and the extra term $4 \pi \rho$ ) are introduced a priori, through equation (18). This allows for a much shorter derivation of the strange term, without any periodicity assumption.

\subsection{Proof of Theorem 2}

Standard estimates show that the sequence $u_{n}$ of solutions of (4) is bounded in $\dot{H}^{1}\left(\mathbb{R}^{3}\right)^{3} \cap$ $L^{6}\left(\mathbb{R}^{3}\right)^{3}$. Let $\varphi \in C_{c}^{\infty}\left(\mathbb{R}^{3}\right)^{3}$ a divergence-free vector field. We introduce the solution $\phi_{n}$ of

$$
-\Delta \phi_{n}+\nabla q_{n}=-6 \pi \rho \varphi, \quad \operatorname{div} \phi_{n}=0 \text { in } \Omega_{n},\left.\quad \phi_{n}\right|_{B_{i}}=\varphi .
$$

Arguing exactly as in Paragraph 3.1, to show the weak convergence of $u_{n}$ to the solution $u$ of (9), it is enough to prove the convergence of $\phi_{n}$ to zero in the sense of distributions. We consider this time the matrix-valued solution $\Phi_{n}$ of

$$
-\Delta \Phi_{n}+\nabla Q_{n}=-6 \pi \rho I, \quad \operatorname{div} \Phi_{n}=0 \text { in } \Omega_{n},\left.\quad \Phi_{n}\right|_{B_{i}}=I,
$$


where $I$ denotes the identity matrix. With $R_{n}=\phi_{n}-\Phi_{n} \varphi$, and $S_{n}=q_{n}-Q_{n} \cdot \varphi$, we find

$$
-\Delta R_{n}+\nabla S_{n}=2 \partial_{i} \Phi_{n} \partial_{i} \varphi+\Phi_{n} \Delta \varphi+(\nabla \varphi) Q_{n}, \quad \operatorname{div} R_{n}=\Phi_{n}: \nabla \varphi \quad \text { in } \Omega_{n},\left.\quad R_{n}\right|_{B_{i}}=0 .
$$

We admit for the time being

Lemma 5. Let $q>3$, and $U$ a smooth bounded domain containing $K$. There exists a family of operators $\mathcal{B}_{n}: L_{0}^{q}\left(\Omega_{n} \cap U\right) \rightarrow H_{0}^{1}\left(\Omega_{n} \cap U\right)$ such that div $\mathcal{B}_{n} h=h$ for all $h \in L_{0}^{q}\left(\Omega_{n} \cap U\right)$ and such that $\left\|\mathcal{B}_{n}\right\|_{\mathcal{L}\left(L_{0}^{q}, H_{0}^{1}\right)}$ is bounded uniformly in $n$.

Let $U$ containing both $K$ and the support of $\varphi$. One can check easily that $\Phi_{n}: \nabla \varphi$ has zero average in both $U$ and $\Omega_{n} \cap U$. Extending $\mathcal{B}_{n}\left(\Phi_{n}: \nabla \varphi\right)$ by zero outside $U$, the field $R_{n}^{\prime}=R_{n}-\mathcal{B}_{n}\left(\Phi_{n}: \nabla \varphi\right)$ satisfies

$$
-\Delta R_{n}^{\prime}+\nabla S_{n}^{\prime}=2 \partial_{i} \Phi_{n} \partial_{i} \varphi+\Phi_{n} \Delta \varphi+(\nabla \varphi) Q_{n}+\Delta \mathcal{B}_{n}\left(\Phi_{n}: \nabla \varphi\right) \quad \text { in } \Omega_{n},
$$

plus divergence-free and homogeneous Dirichlet conditions. By the previous lemma and a standard estimate, if we prove that $\Phi_{n} \rightarrow 0$ strongly in $L_{l o c}^{q}$ for some $q>3$ and that $Q_{n} \rightarrow 0$ strongly in $H^{-1}(U)$ (for some appropriate extension in $\cup_{i} B_{i}$ ), then $R_{n}^{\prime} \rightarrow 0$ strongly in $H_{l o c}^{1}$, and so does $R_{n}$. Eventually, $\phi_{n}$ will go to zero.

Therefore, we decompose $\Phi_{n}=\Phi_{n}^{1}+\Phi_{n}^{2}, Q_{n}=Q_{n}^{1}+Q_{n}^{2}$. This time,

$$
\Phi_{n}^{1}(x)=6 \pi \sum_{i} \mathcal{G}_{S t}\left(n\left(x-x_{i}\right)\right)-6 \pi G_{S t} \star \rho .
$$

Here, $G_{S t}(x)=\frac{1}{8 \pi}\left(\frac{I}{|x|}+\frac{x \otimes x}{|x|^{3}}\right)$ is the kernel of $-\mathbb{P} \Delta$, and for $R_{S t}(x)=\frac{1}{8 \pi}\left(\frac{I}{3|x|^{3}}-\frac{x \otimes x}{|x|^{5}}\right)$, we have set

$$
\mathcal{G}_{S t}(x)=G_{S t}(x)+R_{S t}(x) \quad \text { if }|x| \geq 1, \quad \mathcal{G}_{S t}(x)=\frac{I}{6 \pi} \quad \text { if }|x| \leq 1 .
$$

A tedious calculation shows that

$$
-\Delta \Phi_{n}^{1}+\nabla Q_{n}^{1}=6 \pi\left(\delta_{n}^{1 / n}-\rho\right) I, \quad \operatorname{div} \Phi_{n}^{1} \quad \text { in } \mathbb{R}^{3} .
$$

while

$$
-\Delta \Phi_{n}^{2}+\nabla Q_{2}^{n}=0, \quad \operatorname{div} \Phi_{n}^{2}=0 \quad \text { in } \quad \Omega_{n}
$$

with boundary conditions

$$
\left.\Phi_{n}^{2}\right|_{B_{i}}(x)=-\frac{6 \pi}{n} \sum_{j \neq i} G_{S t}\left(x-x_{j}\right)+6 \pi G_{S t} \star \rho(x)-\frac{6 \pi}{n^{3}} \sum_{j \neq i} R_{S t}\left(x-x_{j}\right), \quad x \in B_{i} .
$$

To estimate $\Phi_{n}^{2}$, we use the following (see below for a proof):

Lemma 6. Under (H1), inequality (22) is true for all (matrix or vector-valued) family $\left(w_{i}\right)_{1 \leq i \leq n}$ in $\prod_{i} W^{1, \infty}\left(B_{i}\right)$ satisfying the compatibility condition $\int_{\partial B_{i}} w_{i} \cdot n=0$ for all $i$, and $u[w]$ the solution of

$$
-\Delta u[w]+\nabla p[w]=0, \quad \operatorname{div} u[w]=0 \quad \text { in } \Omega_{n},\left.\quad u[w]\right|_{B_{i}}=w_{i} \text { for all } i .
$$


Using this lemma and (H2'), we find that $\Phi_{n}^{2}$ converges strongly to zero in $\dot{H}^{1}\left(\mathbb{R}^{3}\right) \cap L^{6}\left(\mathbb{R}^{3}\right)$. Thanks to Lemma 5 , we can then show that for any $p<\frac{3}{2}$, the pressure term $Q_{n}^{2}$, normalized to be mean free in $\Omega_{n} \cap U$ and extended by zero in $\cup_{i} B_{i}$, converges strongly in $L^{p}(U)$. Indeed, for any $q>3$ and any $h \in L^{q}(U)$, denoting $h_{0}=h-f_{\Omega_{n} \cap U} h$, we find

$$
\begin{aligned}
\int_{U} Q_{n}^{2} h & =\int_{\Omega_{n} \cap U} Q_{n}^{2} h_{0}=\int_{\Omega_{n} \cap U} Q_{n}^{2} \operatorname{div} \mathcal{B}_{n} h_{0}=-\int_{\Omega_{n} \cap U} \nabla Q_{n}^{2} \mathcal{B}_{n} h_{0}=-\int_{\Omega_{n} \cap U} \Delta \Phi_{n}^{2} \mathcal{B}_{n} h_{0} \\
& =\int_{\Omega_{n} \cap U} \nabla \psi_{n}^{2} \cdot \nabla \mathcal{B}_{n} h_{0} \leq C\left\|\nabla \Phi_{n}^{2}\right\|_{L^{2}\left(\Omega_{n} \cap U\right)}\left\|\nabla \mathcal{B}_{n} h_{0}\right\|_{L^{2}\left(\Omega_{n} \cap U\right)} \\
& \leq C\left\|\nabla \Phi_{n}^{2}\right\|_{L^{2}\left(\mathbb{R}^{3}\right)}\left\|h_{0}\right\|_{L^{q}(U)} \leq C^{\prime}\left\|\nabla \Phi_{n}^{2}\right\|_{L^{2}\left(\mathbb{R}^{3}\right)}\|h\|_{L^{q}(U)}
\end{aligned}
$$

The strong convergence of $Q_{n}^{2}$ in $L^{p}, p=q^{\prime}$, follows.

We finally have to look at the convergence of $\left(\Phi_{n}^{1}, Q_{n}^{1}\right)$ solving $(25)$. The source $6 \pi\left(\delta_{n}^{1 / n}-\rho\right) I$ is compactly supported, and converges to zero in the sense of measures, in particular weakly in $W^{-1, p}\left(\mathbb{R}^{3}\right)$ for any $p<\frac{3}{2}$. Moreover, for an appropriate normalization of the pressure $Q_{n}^{1}$, we have the estimate

$$
\left\|\nabla \Phi_{n}^{1}\right\|_{L^{p}\left(\mathbb{R}^{3}\right)}+\left\|\Phi_{n}^{1}\right\|_{L^{3 p /(3-p)}\left(\mathbb{R}^{3}\right)}+\left\|Q_{n}^{1}\right\|_{L^{p}\left(\mathbb{R}^{3}\right)} \leq C\left\|6 \pi\left(\delta_{n}^{1 / n}-\rho\right) I\right\|_{W^{-1, p}\left(\mathbb{R}^{3}\right)}
$$

see $\left[17\right.$, Theorem 4.2.2]. This implies that for any $p<\frac{3}{2}, \Phi_{n}^{1} \rightarrow 0$ weakly in $L^{\frac{3 p}{3-p}}\left(\mathbb{R}^{3}\right)$ and strongly in $L_{l o c}^{q}$ for any $q<\frac{3 p}{3-p}$, while $Q_{n}^{1} \rightarrow 0$ weakly in $L^{p}\left(\mathbb{R}^{3}\right)$.

Collecting all previous facts, we see that $\Phi_{n} \rightarrow 0$ strongly in $L_{l o c}^{q}$ for any $q<3$, and $Q_{n} \rightarrow 0$ weakly in $L^{p}(U)$ for any $p<\frac{3}{2}$, therefore strongly in $H^{-1}(U)$. To gain on the exponent $q$ for $\Phi_{n}$ and conclude the proof, it is enough to show that $\nabla \Phi_{n}$ is bounded in $L^{2}\left(\mathbb{R}^{3}\right)$, which implies strong convergence in $L_{l o c}^{q}$ for any $q<6$. Such uniform bound can be obtained easily: by use of standard Bogovski operator, one can find a compactly supported (matrix-valued) $H^{1}$ field $\Phi_{I}$ which is divergence-free and such that $\Phi_{I}=I$ in a neighborhood of $K \supset \cup_{i} B_{i}$. A uniform $L^{2}$ bound on $\nabla\left(\Phi_{n}-\Phi_{I}\right)$ then follows from a standard energy estimate, as $\Phi_{n}-\Phi_{I}$ is zero in the balls.

Proof of Lemma 5. Let $q>3$, and $h \in L_{0}^{q}\left(\Omega_{n} \cap U\right)$. We extend $h$ by zero in $\cup_{i} B_{i}$. First, we introduce a standard Bogovski operator $\mathcal{B}: L^{q}(U) \rightarrow W_{0}^{1, q}(U)$ which is continuous and satisfies $\operatorname{div} \mathcal{B} h=h$. The next step is to find a field $w_{n}=w_{n}[h]$ in $H_{0}^{1}(U)$ such that

$$
\operatorname{div} w_{n}=0 \text { in } U \cap \Omega_{n},\left.\quad w_{n}\right|_{B_{i}}=\left.\mathcal{B} h\right|_{B_{i}}, \quad\left\|w_{n}\right\|_{H_{0}^{1}} \leq C\|\mathcal{B} h\|_{W_{0}^{1, q}}, \quad C \text { independent of } n .
$$

Indeed, $\mathcal{B}_{n} h=\mathcal{B} h-w_{n}$ will have the required properties. As the balls $n B_{i}=B\left(n x_{i}, 1\right)$ have radius 1, application of usual Bogovski operators yield for each $1 \leq i \leq n$ a divergence-free function $W_{i} \in H_{0}^{1}\left(B\left(n x_{i}, 2\right)\right)$ such that

$$
\left.W_{i}\right|_{n B_{i}}=\left.\mathcal{B} h\left(n^{-1} \cdot\right)\right|_{n B_{i}}, \quad\left\|W_{i}\right\|_{H_{0}^{1}\left(B\left(n x_{i}, 2\right)\right)} \leq C\left\|\mathcal{B} h\left(n^{-1} \cdot\right)\right\|_{H^{1}\left(n B_{i}\right)}, \quad C \text { independent of } n .
$$

We set $w_{n}(x)=\sum_{i} W_{i}(n x)$. Clearly, $w_{n}$ is divergence-free, and belongs to $H_{0}^{1}(U)$. Moreover, assumption (H1) implies that the balls $B\left(n x_{i}, 2\right)$ are disjoint, so that $\left.w_{n}\right|_{B_{i}}=\left.W_{i}(n \cdot)\right|_{B_{i}}=$ $\left.\mathcal{B} h\right|_{B_{i}}$, and

$$
\begin{aligned}
\left\|w_{n}\right\|_{H_{0}^{1}(U)}^{2} & \leq C\left\|\nabla w_{n}\right\|_{L^{2}(U)}^{2}=\sum_{i}\left\|\nabla\left(W_{i}(n \cdot)\right)\right\|_{L^{2}\left(B\left(x_{i}, 2 / n\right)\right)}^{2} \\
& \leq C \sum_{i}\left(n^{2}\|\mathcal{B} h\|_{L^{2}\left(B_{i}\right)}^{2}+\|\nabla \mathcal{B} h\|_{L^{2}\left(B_{i}\right)}^{2}\right) \\
& \leq \frac{4 \pi C}{3}\|\mathcal{B} h\|_{L^{\infty}(U)}+C\|\nabla \mathcal{B} h\|_{L^{2}(U)}^{2} \leq C^{\prime}\|\mathcal{B} h\|_{W_{0}^{1, q}(U)} .
\end{aligned}
$$


where the last inequality involves the Sobolev embedding $W^{1, q} \hookrightarrow L^{\infty}$. This ends the proof.

Proof of Lemma 6. By the classical variational characterization of the Stokes solution,

$$
\|\nabla u[w]\|_{L^{2}\left(\mathbb{R}^{3}\right)}^{2} \leq\|\nabla v[w]\|_{L^{2}\left(\mathbb{R}^{3}\right)}^{2}
$$

for any divergence-free vector field $v=v[w]$ such that $\left.v\right|_{B_{i}}=w_{i}$ for all $i$. Hence, it is enough to prove that there exists such a $v$ satisfying the bound (22). One proceeds as in the proof of Lemma 5: one looks for $v$ under the form $v=\sum_{i} W_{i}(n x)$, where $W_{i} \in H_{0}^{1}\left(B\left(n x_{i}, 2\right)\right)$ is provided through the use of Bogovski operator:

$$
\left.W_{i}\right|_{n B_{i}}=\left.w_{i}\left(n^{-1} \cdot\right)\right|_{n B_{i}}, \quad\left\|W_{i}\right\|_{H_{0}^{1}\left(B\left(n x_{i}, 2\right)\right)} \leq C\left\|w_{i}\left(n^{-1} \cdot\right)\right\|_{H^{1}\left(n B_{i}\right)}, \quad C \text { independent of } n .
$$

Estimate (22) follows as in the proof of Lemma 5.

Remark 7. One can extend the result for the Stokes system (4) to the Navier-Stokes system

$$
-\Delta u_{n}+u_{n} \cdot \nabla u_{n}+\nabla p_{n}=g, \quad \operatorname{div} u_{n}=0 \quad \text { in } \Omega_{n},\left.\quad u_{n}\right|_{B_{i, n}}=0, \quad 1 \leq i \leq n .
$$

In such a case, we can not use directly the test function $\varphi-\phi_{n}$, as $\Omega_{n}$ is unbounded : for instance, the extra term $\int_{\mathbb{R}^{3}}\left(u_{n} \cdot \nabla u_{n}\right) \cdot \phi_{n}=-\int_{\mathbb{R}^{3}}\left(u_{n} \otimes u_{n}\right): \nabla \phi_{n}$ due to the nonlinearity is not a priori properly defined. To circumvent this problem, one can use as a test function $\chi\left(\varphi-\phi_{n}\right)=\varphi-\chi \phi_{n}$, where $\chi \in C_{c}^{\infty}\left(\mathbb{R}^{3}\right)$ satisfies $\chi=1$ in a large open ball containing all the balls $B_{i}$ and the support of $\varphi$. Compared to the Stokes case, there are then several extra integrals in the variational formulation:

$$
\begin{aligned}
I_{n} & :=\int_{\mathbb{R}^{3}}\left(u_{n} \otimes u_{n}\right): \nabla\left(\varphi-\chi \phi_{n}\right), \quad J_{n}:=\int_{\mathbb{R}^{3}} \nabla u_{n}:\left(\nabla \chi \otimes \phi_{n}\right)-\left(u_{n} \otimes \nabla \chi\right): \nabla \phi_{n} \\
K_{n} & :=\int_{\mathbb{R}^{3}} p_{n} \nabla \chi \cdot \phi_{n}, \quad L_{n}:=\int_{\mathbb{R}^{3}} q_{n} \nabla \chi \cdot u_{n}, \quad M_{n}:=\int_{\mathbb{R}^{3}}(1-\chi) g \phi_{n}
\end{aligned}
$$

By a simple energy estimate and Sobolev embedding, still valid in the Navier-Stokes case, $u_{n}$ converges to $u$ weakly in $\dot{H}^{1} \cap L^{6}$, thus strongly in $L_{l o c}^{p}$ for any $p<6$. From the proof above, we also have that $\phi_{n}$ converges to zero weakly in $\dot{H}^{1} \cap L^{6}$, thus strongly in $L_{l o c}^{p}$ for any $p<6$. From there, clearly: $I_{n} \rightarrow \int_{\mathbb{R}^{3}}(u \otimes u): \nabla \varphi, \quad J_{n} \rightarrow 0, \quad M_{n} \rightarrow 0$. As regards $K_{n}$, we use the fact that we can choose an open neighborhood $U$ of the support of $\nabla \chi$, independent of $n$, such that

$$
-\Delta u_{n}+\nabla p_{n}=-\operatorname{div}\left(u_{n} \otimes u_{n}\right)+g \quad \text { in } U .
$$

Choosing $p_{n}$ so that it has zero average in $U$, we deduce from standard estimates that

$$
\left\|p_{n}\right\|_{L^{2}(U)} \leq C_{U}\left(\left\|\nabla u_{n}\right\|_{L^{2}(U)}+\left\|u_{n} \otimes u_{n}\right\|_{L^{2}(U)}+\|g\|_{L^{6 / 5}(U)} \leq C^{\prime} .\right.
$$

Using the strong convergence of $\phi_{n}$ in $L_{l o c}^{2}$ and this uniform bound, we get $K_{n} \rightarrow 0$. By inverting the roles of $\left(u_{n}, p_{n}\right)$ and $\left(\phi_{n}, q_{n}\right)$, we get $L_{n} \rightarrow 0$ as well, resulting in the limit system

$$
-\Delta u+u \cdot \nabla u+\nabla p+6 \pi \rho u=g, \quad \operatorname{div} u=0 \text { in } \mathbb{R}^{3} .
$$

Remark 8. The same methodology applies to the system considered in [14]-[23], where the homogeneous Dirichlet condition in (4) is replaced by an inhomogeneous one:

$$
\forall 1 \leq i \leq n,\left.\quad u_{n}\right|_{B_{i, n}}=V_{i, n}
$$

for a collection of constant velocities $\left(V_{i, n}\right)_{1 \leq i \leq n}$ satisfying

$$
\sup _{n} \frac{1}{n} \sum_{i}\left|V_{i, n}\right|^{2}<+\infty, \quad j_{n}:=\frac{1}{n} \sum V_{i, n} \delta_{x_{i, n}} \underset{n \rightarrow \infty}{\longrightarrow} j \quad \text { in the sense of measures. }
$$


To handle this situation, there is one extra step: to show that the solution $v_{n}$ of

$$
-\Delta v_{n}+\nabla q_{n}=-6 \pi j, \quad \operatorname{div} v_{n}=0 \quad \text { in } \Omega_{n},\left.\quad v_{n}\right|_{B_{i, n}}=V_{i, n}
$$

converges to zero weakly in $\dot{H}^{1}\left(\mathbb{R}^{3}\right)^{3} \cap L^{6}\left(\mathbb{R}^{3}\right)^{3}$. Assuming such convergence, one can then notice that $u_{n}-v_{n}$ satisfies a system of type (4), with source term $g+6 \pi j$ replacing $j$. By Theorem 2, the weak limit of $u_{n}$, which coincides with the weak limit of $u_{n}-v_{n}$, satisfies

$$
-\Delta u+\nabla p+6 \pi u=g+6 \pi j, \quad \operatorname{div} u \quad \text { in } \mathbb{R}^{3} .
$$

To prove the convergence of $v_{n}$ to zero, we mimic what was done for $\Phi_{n}$, solution of $(24)$. Just as we decomposed $\Phi_{n}=\Phi_{n}^{1}+\Phi_{n}^{2}$, we write $v_{n}=v_{n}^{1}+v_{n}^{2}$, with approximate solution

$$
v_{n}^{1}:=6 \pi \sum_{i} \mathcal{G}_{S t}\left(n\left(x-x_{i}\right)\right) V_{i, n}-6 \pi G_{S t} \star j .
$$

Weak convergence of $v_{n}^{1}$ to zero comes from the convergence of $j_{n}$ to $j$, while the remainder $v_{2}^{n}$ goes strongly to zero in $H_{l o c}^{1}$ under the assumption

$$
\begin{aligned}
\limsup _{n \rightarrow+\infty} \sum_{i} \int_{B_{i}}( & n^{2}\left|\frac{1}{n} \sum_{j \neq i} G_{S t}\left(x-x_{j}\right) V_{j, n}-\int_{\mathbb{R}^{3}} G_{S t}(x-y) j(y) d y\right|^{2}+\frac{1}{n^{2}}\left|\sum_{j \neq i} \nabla G_{S t}\left(x-x_{j}\right) V_{j, n}\right|^{2} \\
& \left.+\frac{1}{n^{4}}\left|\sum_{j \neq i} R_{S t}\left(x-x_{j}\right) V_{j, n}\right|^{2}+\frac{1}{n^{6}}\left|\sum_{j \neq i} \nabla R_{S t}\left(x-x_{j}\right) V_{j, n}\right|^{2}\right) d x=0
\end{aligned}
$$

which is similar to (H2'), and holds in classical settings, see Section 4 for discussion of (H2').

\subsection{Proof of Theorem 3}

We remind that here, $r_{n}$ is of order $n^{-1 / 3}$, namely with a volume fraction $\lambda=n \frac{4}{3} \pi r_{n}^{3}$ positive and independent of $n$. As in other examples, the energy estimate and the Sobolev imbedding give that the sequence $\left(u_{n, \lambda}\right)_{n \in \mathbb{N}}$ is bounded in $\dot{H}^{1}\left(\mathbb{R}^{3}\right) \cap L^{6}\left(\mathbb{R}^{3}\right)$. After extraction, it converges weakly to some $u_{\lambda}$. Let $\varphi \in \dot{H}^{1}\left(\mathbb{R}^{3}\right) \cap L^{6}\left(\mathbb{R}^{3}\right) \cap C^{\infty}\left(\mathbb{R}^{3}\right)$. We consider $\phi_{n, \lambda}$ satisfying

$-\Delta \phi_{n, \lambda}=\operatorname{div}\left(3 \lambda\left|K_{\rho}\right| \rho \nabla \varphi\right)$ in $\Omega_{n}, \int_{\partial B_{i}} \partial_{\nu} \phi_{n, \lambda}=-\int_{\partial B_{i}} 3 \lambda\left|K_{\rho}\right| \rho \partial_{\nu} \varphi,\left.\phi_{n, \lambda}\right|_{B_{i}}=\left.\varphi\right|_{B_{i}}+$ cst, $\forall i$.

Testing $\varphi-\phi_{n, \lambda}$ in (5), we obtain after a few integrations by parts

$$
\int_{\mathbb{R}^{3}} \nabla u_{n, \lambda} \cdot\left(1+3 \lambda\left|K_{\rho}\right| \rho\right) \nabla \varphi=\int_{\mathbb{R}^{3}} g \varphi-\int_{\mathbb{R}^{3}} g \phi_{n, \lambda} .
$$

As $n$ goes to infinity, the left-hand side converges to $\int_{\mathbb{R}^{3}} \nabla u_{\lambda} \cdot(1+3 \lambda f) \nabla \varphi$, which implies that the last term at the right-hand side, that can be written in the abstract form $\left\langle R_{n, \lambda}, \varphi\right\rangle$ for an element $R_{n, \lambda}$ in the dual of $\dot{H}^{1}\left(\mathbb{R}^{3}\right) \cap L^{6}\left(\mathbb{R}^{3}\right)$, converges to some $\left\langle R_{\lambda}, \varphi\right\rangle$, with $R_{\lambda}$ in this same dual. To prove the theorem, it is enough to show that

$$
\limsup _{n \rightarrow+\infty}\left|\int_{\mathbb{R}^{3}} g \phi_{n, \lambda}\right| \leq \lambda \eta(\lambda)\|\nabla \varphi\|_{L^{\infty}}, \quad \eta(\lambda) \underset{\lambda \rightarrow 0}{\longrightarrow} 0 .
$$

Let us stress that restricting to smooth test functions $\varphi$ instead of Lipschitz is no problem: indeed, if (29) holds for smooth functions, we can apply it to $\rho_{\delta} \star \varphi$ with $\rho_{\delta}$ a mollifier. We deduce

$$
\left\langle R_{\lambda}, \rho_{\delta} \star \varphi\right\rangle \leq \lambda \eta(\lambda)\left\|\nabla \rho_{\delta} \star \varphi\right\|_{L^{\infty}} \leq \lambda \eta(\lambda)\|\nabla \varphi\|_{L^{\infty}}
$$


As $\rho_{\delta} \star \varphi$ converges in $\dot{H}^{1}\left(\mathbb{R}^{3}\right) \cap L^{6}\left(\mathbb{R}^{3}\right)$, the l.h.s. converges to $\left\langle R_{\lambda}, \varphi\right\rangle$, which yields (16). We split $\phi_{n, \lambda}=\phi_{n}^{1}+\phi_{n}^{2}$, with

$$
\phi_{n}^{1}=3 \lambda\left|K_{\rho}\right| V \star(\rho \nabla \varphi)-4 \pi r_{n} \sum_{i} \nabla \varphi\left(x_{i}\right) \cdot \mathcal{V}\left(\frac{x-x_{i}}{r_{n}}\right),
$$

where $V(x)=\nabla \frac{1}{4 \pi|x|}, \mathcal{V}(x)=V(x)$ for $|x| \geq 1, \mathcal{V}(x)=-\frac{x}{4 \pi}$ for $|x| \leq 1$.

We compute $\Delta \mathcal{V}=\frac{3}{4 \pi} x s^{1}$, with $s^{\eta}$ the surface measure on the sphere of radius $\eta$. Hence,

$$
\begin{aligned}
-\Delta \phi_{n}^{1} & =\operatorname{div}\left(3 \lambda\left|K_{\rho}\right| \rho \nabla \varphi\right)+\frac{3}{r_{n}} \sum_{i} \nabla \varphi\left(x_{i}\right) \cdot\left(x-x_{i}\right) s^{r_{n}}\left(x-x_{i}\right) \\
& =\operatorname{div}\left(3 \lambda\left|K_{\rho}\right| \rho \nabla \varphi\right)-3 \sum_{i} \nabla \varphi\left(x_{i}\right) \cdot \nabla 1_{B_{i}} \quad \text { in } \mathbb{R}^{3} \\
& =\operatorname{div}\left(3 \lambda\left|K_{\rho}\right| \rho \nabla \varphi-3 \lambda\left|K_{\rho}\right|\left(\frac{1}{n} \sum_{i} \frac{1}{\left|B_{i}\right|} 1_{B_{i}} \nabla \varphi\left(x_{i}\right)\right)\right) \quad \text { in } \mathbb{R}^{3} .
\end{aligned}
$$

One also checks that $\int_{\partial B_{i}} \partial_{\nu} \phi_{n}^{1}=-\int_{\partial B_{i}} 3 \lambda \rho \partial_{\nu} \varphi$. We further decompose $\phi_{n}^{2}=\psi_{n}^{2}+\tilde{\psi}_{n}^{2}$, with

$$
-\Delta \psi_{n}^{2}=-\Delta \tilde{\psi}_{n}^{2}=0, \quad \int_{\partial B_{i}} \partial_{\nu} \psi_{n}^{2}=\int_{\partial B_{i}} \partial_{\nu} \tilde{\psi}_{n}^{2}=0
$$

and for all $i$ :

$$
\begin{aligned}
& \left.\psi_{n}^{2}\right|_{B_{i}}(x)=-3 \lambda\left|K_{\rho}\right| V \star(\rho \nabla \varphi)(x)+4 \pi r_{n} \sum_{j \neq i} \nabla \varphi\left(x_{j}\right) \cdot V\left(\frac{x-x_{j}}{r_{n}}\right)+c s t \\
& \left.\tilde{\psi}_{n}^{2}\right|_{B_{i}}(x)=\varphi(x)-\nabla \varphi\left(x_{i}\right) \cdot\left(x-x_{i}\right)+c s t .
\end{aligned}
$$

By classical variational characterization, the function $\psi_{n}^{2}$, resp. $\tilde{\psi}_{n}^{2}$, minimizes the Dirichlet integral among all functions $\psi \in \dot{H}^{1}\left(\mathbb{R}^{3}\right)$ satisfying $\left.\psi\right|_{B_{i}}=\left.\psi_{n}^{2}\right|_{B_{i}}+c_{i}$, resp. $\left.\psi\right|_{B_{i}}=\left.\tilde{\psi}_{n}^{2}\right|_{B_{i}}+c_{i}$, on $B_{i}$, for some $c_{i} \in \mathbb{R}$, for all $i$. From there, one can proceed as in the proof of $(22), c f$. Lemma 6 , and show that under (A1):

$$
\left\|\nabla \psi_{n}^{2}\right\|_{L^{2}\left(\mathbb{R}^{3}\right)}^{2} \leq C \sum_{i} \int_{B_{i}}\left|\nabla \psi_{n}^{2}\right|^{2}, \quad\left\|\nabla \tilde{\psi}_{n}^{2}\right\|_{L^{2}\left(\mathbb{R}^{3}\right)}^{2} \leq C \sum_{i} \int_{B_{i}}\left|\nabla \tilde{\psi}_{n}^{2}\right|^{2}
$$

note that, in contrast to (22), the right-hand side involves only gradients, consistently with the additional degree of freedom provided by possible addition of constants $c_{i}$ on $B_{i}$. In the case of $\tilde{\psi}_{n}^{2}$, we get

$$
\left\|\nabla \tilde{\psi}_{n}^{2}\right\|_{L^{2}\left(\mathbb{R}^{3}\right)}^{2} \leq C\left\|\nabla^{2} \varphi\right\|_{L^{\infty}} \sum_{i} \int_{B_{i}}\left|x-x_{i}\right|^{2} d x=O\left(n^{-2 / 3}\right) .
$$

As regards $\psi_{n}^{2}$, we compute

$$
\begin{aligned}
\left.\nabla \psi_{n}^{2}\right|_{B_{i}}(x) & =-3 \lambda\left|K_{\rho}\right| \int \rho(y) \nabla V(x-y) \nabla \varphi(y) d y+\frac{3 \lambda\left|K_{\rho}\right|}{n} \sum_{j \neq i} \nabla V\left(x-x_{j}\right) \nabla \varphi\left(x_{j}\right) \\
& =-b(x)+a_{i}(x),
\end{aligned}
$$

where $\nabla V=-\frac{1}{4 \pi} \nabla \frac{x}{|x|^{3}}$ is a Calderon-Zygmund kernel. Hence, $b$ satisfies for all $q>1$ :

$$
\|b\|_{L^{q}\left(\mathbb{R}^{3}\right)} \leq C_{q} \lambda\|\rho \nabla \varphi\|_{L^{q}\left(\mathbb{R}^{3}\right)} \leq C_{q} \lambda\|\rho \nabla \varphi\|_{L^{q}(K)}
$$


It follows by Hölder inequality that for all $q>2$

$$
\sum_{i}\|b\|_{L^{2}\left(B_{i}\right)}^{2}=\|b\|_{L^{2}\left(\cup B_{i}\right)}^{2} \leq \lambda^{\frac{q-2}{q}}\|b\|_{L^{q}\left(\mathbb{R}^{3}\right)}^{2} \leq C \lambda^{3-\frac{2}{q}}\|\nabla \varphi\|_{L^{\infty}}^{2} .
$$

Eventually, by (31), we find for all $\varepsilon>0$ :

$$
\begin{aligned}
\limsup _{n \rightarrow+\infty}\left\|\nabla \psi_{n}^{2}\right\|_{L^{2}\left(\mathbb{R}^{3}\right)}^{2} & \leq C_{\varepsilon} \lambda^{3-\varepsilon}\|\nabla \varphi\|_{L^{\infty}}^{2}+\limsup _{n \rightarrow+\infty} \sum_{i}\left\|a_{i}\right\|_{L^{2}\left(B_{i}\right)}^{2} \\
& \leq\left(C_{\varepsilon} \lambda^{3-\varepsilon}+\eta(\lambda) \lambda^{2}\right)\|\nabla \varphi\|_{L^{\infty}\left(\mathbb{R}^{3}\right)}^{2}, \quad \eta(\lambda) \underset{\lambda \rightarrow 0}{\longrightarrow} 0
\end{aligned}
$$

where the last inequality comes from assumption (A2). Together with (32), we deduce

$$
\limsup _{n \rightarrow+\infty}\left\|\nabla \phi_{n}^{2}\right\|_{L^{2}\left(\mathbb{R}^{3}\right)} \leq \eta(\lambda) \lambda\|\nabla \varphi\|_{L^{\infty}}, \quad \eta(\lambda) \underset{\lambda \rightarrow 0}{\longrightarrow} 0 .
$$

To show (29) and conclude in this way the proof of the theorem, it is enough to show that $\phi_{n}^{1}$ goes weakly to zero as $n \rightarrow+\infty$ in $L^{6}\left(\mathbb{R}^{3}\right)$. Standard estimates on $\phi-\phi_{n, \lambda}$ show that $\phi_{n, \lambda}$, and from there $\phi_{n}^{1}$, is bounded uniformly in $n$ in $\dot{H}^{1}\left(\mathbb{R}^{3}\right) \cap L^{6}\left(\mathbb{R}^{3}\right)$, so that convergence to zero in the sense of distributions is enough. It follows from a duality argument: for $h$ any smooth and compactly supported function, we introduce $H$ the solution of $\Delta H=h$ in $\mathbb{R}^{3}$, and thanks to $(30)$, we write

$$
\left\langle\phi_{n}^{1}, h\right\rangle=\left\langle\Delta \phi_{n}^{1}, H\right\rangle=3 \lambda\left|K_{\rho}\right|\left\langle\rho \nabla \varphi-\frac{1}{n} \sum \frac{1}{\left|B_{i}\right|} 1_{B_{i}} \nabla \varphi\left(x_{i}\right), \nabla H\right\rangle
$$

which is easily seen to go to zero as $n \rightarrow+\infty$, by (H0).

\section{Further discussion of the hypotheses}

We conclude this paper with further comments on the assumptions of our theorems. First, we show that the assumptions (H1) and (H2) in Theorem 1 are implied by the conditions

$$
\begin{gathered}
\lim _{n \rightarrow+\infty} n d_{n}=+\infty, \quad d_{n}=\inf _{i \neq j}\left|x_{i}-x_{j}\right| \\
\lim _{n \rightarrow+\infty} \frac{1}{n} \sum_{i}\left(\frac{1}{n} \sum_{j \neq i} \frac{1}{\left|x_{i}-x_{j}\right|}-\int_{\mathbb{R}^{3}} \frac{\rho(y)}{\left|x_{i}-y\right|} d y\right)^{2}=0
\end{gathered}
$$

Clearly, $\left(\mathrm{H} 1^{\sharp}\right)$ is stronger than (H1). Then, (H2) follows from $\left(\mathrm{H} 2^{\sharp}\right)$ if we prove that

$$
\begin{aligned}
\lim _{n} \sum_{i} \int_{B_{i}}\left(\left|\sum_{j \neq i}\left(G\left(x-x_{j}\right)-G\left(x_{i}-x_{j}\right)\right)\right|^{2}\right. \\
\left.\quad+n^{2}\left|\int_{\mathbb{R}^{3}} G(x-y) \rho(y) d y-\int_{\mathbb{R}^{3}} G\left(x_{i}-y\right) \rho(y) d y\right|^{2}+\left|\frac{1}{n} \sum_{j \neq i} \nabla G\left(x-x_{j}\right)\right|^{2}\right) d x=0 .
\end{aligned}
$$

We focus on the first term, as the second one is simpler and the third one similar. We write:

$$
\begin{aligned}
& \sum_{i} \int_{B_{i}}\left|\sum_{j \neq i}\left(G\left(x-x_{j}\right)-G\left(x_{i}-x_{j}\right)\right)\right|^{2} \\
\leq & C \sum_{i} \int_{B_{i}}\left|\sum_{j \neq i} \sup _{z \in\left[x_{i}, x\right]}\right| \nabla G\left(z-x_{j}\right)|| x-x_{i}||^{2} \\
\leq & \frac{C^{\prime}}{n^{5}} \sum_{i}\left|\sum_{j \neq i} \frac{1}{\left|x_{i}-x_{j}\right|^{2}}\right|^{2} \leq \frac{C^{\prime}}{\left(n d_{n}\right)^{2}} \frac{1}{n} \sum_{i}\left|\frac{1}{n} \sum_{j \neq i} \frac{1}{\left|x_{i}-x_{j}\right|}\right|^{2} \leq \frac{C^{\prime \prime}}{\left(n d_{n}\right)^{2}}
\end{aligned}
$$


where we deduced from $\left(\mathrm{H} 2^{\sharp}\right)$ that $\frac{1}{n} \sum_{i}\left|\frac{1}{n} \sum_{j \neq i} \frac{1}{\left|x_{i}-x_{j}\right|}\right|^{2}$ is bounded uniformly in $n$. Convergence to zero follows then from $\left(\mathrm{H} 1^{\sharp}\right)$.

Note that $\left(\mathrm{H} 2^{\sharp}\right)$ is in the same spirit as the hypothesis (12) used in [34], although a bit stronger. Both $\left(\mathrm{H} 1^{\sharp}\right)$ and $\left(\mathrm{H} 2^{\sharp}\right)$ apply to many contexts. First, they are satisfied when the points are well-separated, $c f$. (11). Let us deduce $\left(\mathrm{H} 2^{\sharp}\right)$ from (11). For all $j$, we set $\mathcal{B}_{j}=B\left(y_{j}, \frac{c}{2} n^{-1 / 3}\right)$, where $c$ is the constant appearing in (11). As $\frac{1}{|x|}$ is harmonic, we apply the mean value formula to write for any $i$ :

$$
\begin{aligned}
\frac{1}{n} \sum_{j \neq i} \frac{1}{\left|x_{i}-x_{j}\right|} & =\frac{1}{n} \sum_{j \neq i} \frac{1}{\left|\mathcal{B}_{j}\right|} \int_{\mathcal{B}_{j}} \frac{1}{\left|x_{i}-y\right|} d y=\frac{1}{n} \sum_{j} \frac{1}{\left|\mathcal{B}_{j}\right|} \int_{\mathcal{B}_{j}} \frac{1}{\left|x_{i}-y\right|} d y+O\left(n^{-2 / 3}\right) \\
& =\int_{\mathbb{R}^{3}} \frac{1}{\left|x_{i}-y\right|} g_{n}(y) d y+O\left(n^{-2 / 3}\right)
\end{aligned}
$$

where $\rho_{n}(y)=\frac{1}{n} \sum_{j} \frac{1}{\left|\mathcal{B}_{j}\right|} 1_{B_{j}}(y)$. By $\left(\mathrm{H} 2^{\sharp}\right)$, the sequence $\left(\rho_{n}\right)_{n \in \mathbb{N}}$ is bounded in $L^{\infty}$, and by (H0) is easily seen to converge weakly $*$ to $\rho$. Hence, for all $x \in K, f_{n}(x)=\int_{\mathbb{R}^{3}} \frac{1}{|x-y|} \rho_{n}(y) d y$ converges to $f(x)=\int_{\mathbb{R}^{3}} \frac{1}{|x-y|} \rho(y) d y$. It is also easily seen that $\left(f_{n}\right)$ is equicontinuous over $K$, from which we deduce that $\sup _{K}\left|f_{n}-f\right| \rightarrow 0$, and from there $\left(\mathrm{H} 2^{\sharp}\right)$.

The convergences in $\left(\mathrm{H} 1^{\sharp}\right)$ and $\left(\mathrm{H} 2^{\sharp}\right)$ hold also in probability when the $x_{i}$ are random i.i.d variables with law $\rho$. More precisely, it is well-known that in such setting, $n^{\alpha} d_{n} \rightarrow+\infty$ in probability for any $\alpha>\frac{2}{3}$, which is of course stronger than $\left(\mathrm{H} 1^{\sharp}\right)$. Moreover,

$$
\lim _{n \rightarrow+\infty} \mathbb{E} \frac{1}{n} \sum_{i}\left(\frac{1}{n} \sum_{j \neq i} \frac{1}{\left|x_{i}-x_{j}\right|}-\int_{\mathbb{R}^{3}} \frac{\rho(y)}{\left|x_{i}-y\right|} d y\right)^{2}=0
$$

which implies again convergence in probability. To establish this last limit is very classical: we write

$$
\begin{aligned}
& \mathbb{E}\left(\frac{1}{n} \sum_{j \neq i} \frac{1}{\left|x_{i}-x_{j}\right|}-\int_{\mathbb{R}^{3}} \frac{\rho(y)}{\left|x_{i}-y\right|} d y\right)^{2}=\mathbb{E}\left(\frac{1}{n} \sum_{j \neq i}\left(\frac{1}{\left|x_{i}-x_{j}\right|}-\int_{\mathbb{R}^{3}} \frac{\rho(y)}{\left|x_{i}-y\right|} d y\right)\right)^{2}+O\left(n^{-2}\right) \\
& =\frac{1}{n^{2}} \sum_{j, j^{\prime} \neq i} \mathbb{E} X_{i j} X_{i j^{\prime}}+O\left(n^{-2}\right)=\frac{1}{n^{2}} \sum_{j \neq j^{\prime} \neq i} \mathbb{E} X_{i j} X_{i j^{\prime}}+O\left(n^{-1}\right)
\end{aligned}
$$

where $X_{i j}:=\frac{1}{\left|x_{i}-x_{j}\right|}-\int_{\mathbb{R}^{3}} \frac{\rho(y)}{\left|x_{i}-y\right|} d x$ has mean zero and variance

$$
\mathbb{E} X_{i j}^{2}=\int \rho(x) \rho(z)\left|\frac{1}{|x-z|}-\int \frac{\rho(y)}{|x-y|} d y\right|^{2} d x d z .
$$

Eventually, for $j \neq j^{\prime} \neq i$, we compute using the independance of the $x_{k}$ 's:

$$
\begin{aligned}
\mathbb{E} X_{i j} X_{i j^{\prime}} & =\int_{\left(\mathbb{R}^{3}\right)^{3}} \rho(z) \rho(x) \rho\left(x^{\prime}\right)\left(\frac{1}{|z-x|}-\int_{\mathbb{R}^{3}} \frac{\rho(y)}{|z-y|} d y\right)\left(\frac{1}{\left|z-x^{\prime}\right|}-\int \frac{\rho\left(y^{\prime}\right)}{\left|z-y^{\prime}\right|} d y^{\prime}\right) d z d x d x^{\prime} \\
& =\int_{\mathbb{R}^{3}} \rho(z)\left(\int_{\mathbb{R}^{3}} \frac{\rho(x)}{|z-x|} d x-\int_{\mathbb{R}^{3}} \frac{\rho(y)}{|z-y|} d y\right)\left(\int_{\mathbb{R}^{3}} \frac{\rho\left(x^{\prime}\right)}{\left|z-x^{\prime}\right|} d x^{\prime}-\int_{\mathbb{R}^{3}} \frac{\rho\left(y^{\prime}\right)}{\left|z-y^{\prime}\right|} d y^{\prime}\right) d z=0 .
\end{aligned}
$$

Hence, $\left(\mathrm{H} 1^{\sharp}\right)-\left(\mathrm{H} 2^{\sharp}\right)$ holds in probability when the $x_{i}$ are i.i.d. random variables. We remind that a sequence is converging in probability if and only if any subsequence has itself a subsequence that converges almost surely. Using this characterization, and applying the proof of Theorem 1, we find that $u_{n}$ converges to $u$ solution of (8) in probability, for any distance metrizing the weak topology of the ball of $\dot{H}^{1} \cap L^{6}$ to which all $u_{n}$ belong. 
Similar considerations apply to the Stokes case. We leave to the reader to check that assumptions (H1)-(H2') are implied by $\left(\mathrm{H} 1^{\sharp}\right)-\left(\mathrm{H} 2^{\prime} \sharp\right)$, with

$$
\lim _{n \rightarrow+\infty} \frac{1}{n} \sum_{i}\left(\frac{1}{n} \sum_{j \neq i} \frac{\left(x_{i}-x_{j}\right) \otimes\left(x_{i}-x_{j}\right)}{\left|x_{i}-x_{j}\right|^{3}}-\int_{\mathbb{R}^{3}} \frac{\left(x_{i}-y\right) \otimes\left(x_{i}-y\right)}{\left|x_{i}-y\right|^{3}} \rho(y) d y\right)^{2}=0 .
$$

These stronger hypotheses are again verified under the assumption (11), or in the i.i.d. case. To this respect, the only real change is in showing that (11) implies (H2 $\left.{ }^{\prime \sharp}\right)$. We use this time that $\frac{x}{|x|^{3}}=-\nabla \frac{1}{|x|}$ is harmonic and obtain

$$
\begin{aligned}
\frac{1}{n} \sum_{j \neq i} \frac{\left(x_{i}-x_{j}\right) \otimes\left(x_{i}-x_{j}\right)}{\left|x_{i}-x_{j}\right|^{3}} & =\frac{1}{n} \sum_{j \neq i} \frac{1}{\left|\mathcal{B}_{j}\right|} \int_{\mathcal{B}_{j}} \frac{x_{i}-y}{\left|x_{i}-y\right|^{3}} \otimes\left(x_{i}-x_{j}\right) d y \\
& =\int_{\mathbb{R}^{3}} \frac{x_{i}-y}{\left|x_{i}-y\right|^{3}} \otimes g_{n}\left(x_{i}, y\right) d y+O\left(n^{-2 / 3}\right)
\end{aligned}
$$

where $g_{n}(x, y)=\frac{1}{n} \sum_{j} \frac{1}{\left|\mathcal{B}_{j}\right|}\left(x-x_{j}\right) 1_{B_{j}}(y)$ converges for all $x$ weakly $*$ to $g(x, y)=(x-y) \rho(y)$ in $L_{y}^{\infty}$. Thus, $f_{n}(x)=\int_{\mathbb{R}^{3}} \frac{x-y}{|x-y|^{3}} \otimes g_{n}(x, y) d y$ converges to $f(x)=\int_{\mathbb{R}^{3}} \frac{x-y}{|x-y|^{3}} \otimes g(x, y) d y$ and one can check that $f_{n}$ is equicontinuous over $K$. We conclude as in the case of the Laplacian.

We now turn to the discussion of assumptions (A1)-(A2). We shall see that they are satisfied under the strong assumption (11) on the minimal distance. The point is to check (A2). For all $i$ and smooth $\varphi$, we set $R_{i}(x)=\frac{1}{n} \sum_{j \neq i} \nabla V\left(x-x_{j}\right) \nabla \varphi\left(x_{j}\right)$. We shall prove the stronger statement:

$$
\limsup _{n} \sum_{i}\left\|R_{i}\right\|_{L^{2}\left(B_{i}\right)}^{2} \leq C \lambda^{1-\varepsilon}\|\nabla \varphi\|_{L^{\infty}}^{2} \quad \forall \varepsilon>0
$$

We have for all $x \in B_{i}$,

$$
\begin{aligned}
\left|R_{i}(x)\right| & \leq\left|R_{i}(x)-R_{i}\left(x_{i}\right)\right|+\left|R_{i}\left(x_{i}\right)\right| \leq\left\|\nabla R_{i}\right\|_{L^{\infty}}\left|x-x_{i}\right|+\left|R_{i}\left(x_{i}\right)\right| \\
& \leq \frac{C}{n} \sum_{j \neq i} \frac{r_{n}}{\left|x_{i}-x_{j}\right|^{4}}|| \nabla \varphi \|_{L^{\infty}}+\left|R_{i}\left(x_{i}\right)\right|
\end{aligned}
$$

By assumption (11), setting $y_{k}=n^{1 / 3} x_{k}$, we find

$$
\frac{1}{n} \sum_{j \neq i} \frac{r_{n}}{\left|x_{i}-x_{j}\right|^{4}}\|\nabla \varphi\|_{L^{\infty}} \leq r_{n} n^{1 / 3} \sum_{j \neq i} \frac{1}{\left|y_{i}-y_{j}\right|^{4}}\|\nabla \varphi\|_{L^{\infty}} \leq C \lambda^{1 / 3}\|\nabla \varphi\|_{L^{\infty}} .
$$

Hence,

$$
\sum_{i}\left\|a_{i}\right\|_{L^{2}\left(B_{i}\right)}^{2} \leq C \lambda^{\frac{5}{3}}\|\nabla \varphi\|_{L^{\infty}}^{2}+r_{n}^{3} \sum_{i}\left|R_{i}\left(x_{i}\right)\right|^{2} .
$$

From a slight variation of [18, Lemma 2.4], see also [24], we get for all $q \geq 2$, and $p$ the conjugate exponent of $q$ :

$$
\sum_{i}\left|R_{i}\left(x_{i}\right)\right|^{q} \leq \frac{C}{\lambda^{q}} \lambda^{q / p} \sum_{i}\left|\nabla \varphi\left(x_{i}\right)\right|^{q}=\frac{C}{\lambda} \sum_{i}\left|\nabla \varphi\left(x_{i}\right)\right|^{q}
$$

Then, by Hölder inequality:

$$
r_{n}^{3} \sum_{i}\left|R_{i}\left(x_{i}\right)\right|^{2} \leq r_{n}^{3} n^{\frac{q-2}{q}}\left(\sum_{i}\left|R_{i}\left(x_{i}\right)\right|^{q}\right)^{\frac{2}{q}} \leq C r_{n}^{3} n^{\frac{q-2}{q}}\left(\frac{1}{\lambda} \sum_{i}\left|\nabla \varphi\left(x_{i}\right)\right|^{q}\right)^{\frac{2}{q}} \leq C \lambda^{1-\frac{2}{q}}\|\nabla \varphi\|_{L^{\infty}}^{2}
$$

The result follows. 
Eventually, it is interesting to understand the meaning of (A2) when the centers $x_{i, n}$ of the balls are given by a stationary point process $[12,13]$. More precisely, we will discuss under what conditions on the process we have: for all smooth $\varphi$,

$$
\limsup _{n \rightarrow+\infty} \frac{1}{n^{2}} \sum_{i} \int_{B_{i}}\left|\sum_{j \neq i} \nabla V\left(x-x_{j}\right) \nabla \varphi\left(x_{j}\right)\right|^{2} d x \rightarrow 0, \quad \lambda \rightarrow 0 .
$$

This assumption is slightly weaker than (A2): it only implies that for $\varphi \in \dot{H}^{1} \cap L^{6} \cap C^{\infty}$, $\left\langle R_{\lambda}, \varphi\right\rangle=o(\lambda)$, with $R_{\lambda}$ the remainder in (15).

Let $\Lambda$ a random point process on a probability space $\Omega$. In particular, for $\omega \in \Omega, \Lambda(\omega)$ is a discrete subset of $\mathbb{R}^{3}$. We assume that the process is stationary, of mean intensity $\lambda_{0}$, and ergodic. Note that we allow $\lambda_{0}$ to depend on $\lambda$. Then, given a small parameter $\varepsilon$, we set $\left\{x_{1, n}, \ldots, x_{n, n}\right\}=\varepsilon \Lambda \cap \mathcal{O}$, where the labeling of the centers is arbitrary. Note that $n$ depends on $\varepsilon$, and is random: by the ergodic theorem, $n \varepsilon^{3} \rightarrow \lambda_{0}|\mathcal{O}|$ almost surely as $\varepsilon \rightarrow 0$. It implies that $r_{n} \varepsilon^{-1} \rightarrow\left(\frac{3}{4 \pi} \lambda / \lambda_{0}\right)^{1 / 3}$ almost surely as $\varepsilon \rightarrow 0$. It allows to reformulate condition $\left(\mathrm{A} 2_{b}\right)$ : for all smooth $\varphi$,

$$
\limsup _{\varepsilon \rightarrow 0} \frac{\varepsilon^{6}}{\lambda_{0}^{2}} \sum_{i} \int_{B_{i}}\left|\sum_{j \neq i} \nabla V\left(x-x_{j}\right) \nabla \varphi\left(x_{j}\right)\right|^{2} d x \rightarrow 0, \quad \lambda \rightarrow 0 .
$$

We want to understand under what conditions

$$
\mathbb{E} \limsup _{\varepsilon \rightarrow 0} \frac{\varepsilon^{6}}{\lambda_{0}^{2}} \sum_{i} \int_{B_{i}}\left|\sum_{j \neq i} \nabla V\left(x-x_{j}\right) \nabla \varphi\left(x_{j}\right)\right|^{2} d x \rightarrow 0, \quad \lambda \rightarrow 0
$$

which implies $\left(\mathrm{A} 2_{b}\right)$ in probability. First, we want to reverse the limsup and the expectation. This will follow from the dominated convergence theorem if we show an $L^{\infty}$ bound on

$$
I_{\varepsilon}=\frac{\varepsilon^{6}}{\lambda_{0}^{2}} \sum_{i} \int_{B_{i}}\left|\sum_{j \neq i} \nabla V\left(x-x_{j}\right) \nabla \varphi\left(x_{j}\right)\right|^{2} d x
$$

that is uniform in $\varepsilon$ and $\omega$ (but not necessarily on $\lambda$ ). We claim that such bound holds if for instance the process satisfies the condition

$$
\left|y_{i}-y_{j}\right| \geq c\left(\lambda / \lambda_{0}\right)^{1 / 3}, \quad c>2 \quad \forall i \neq j
$$

which ensures that for $\varepsilon$ small enough, assumption (A1) holds. Indeed, using that $\nabla V$ is a harmonic function, we can write

$$
I_{\varepsilon}=\frac{\varepsilon^{6}}{\lambda_{0}^{2}} \sum_{i} \int_{B_{i}}\left|\sum_{j \neq i} f_{B_{j}} \nabla V\left(x-x_{j}\right) d y \nabla \varphi\left(x_{j}\right)\right|^{2} d x=\frac{\varepsilon^{6}}{\lambda_{0}^{2} r_{n}^{6}} \sum_{i} \int_{B_{i}}\left|\nabla^{2} \Delta^{-1} \sum_{j \neq i} 1_{B_{j}} \nabla \varphi\left(x_{j}\right)\right|^{2} d x .
$$

Note that $\left|\frac{\varepsilon^{6}}{r_{n}^{6}}\right| \leq \frac{C_{0}}{\lambda^{2}}$ for an absolute constant $C_{0}$. We also remind that $\lambda_{0}$ possibly depends on $\lambda$. Hence, we find that

$$
I_{\varepsilon} \leq C_{\lambda} \sum_{i} \int_{B_{i}}\left|\nabla^{2} \Delta^{-1} 1_{B_{i}} \nabla \varphi\left(x_{i}\right)\right|^{2}+C_{\lambda} \int_{\mathbb{R}^{3}}\left|\nabla^{2} \Delta^{-1} \sum_{j} 1_{B_{j}} \nabla \varphi\left(x_{j}\right)\right|^{2} \leq C_{\lambda}^{\prime}
$$

where we use the well-know fact that $\nabla^{2} \Delta^{-1} 1_{B_{i}}$ is bounded in $L^{\infty}$ to control the first term, while we use the continuity of $\nabla^{2} \Delta^{-1}$ over $L^{2}$ and the fact that the balls $B_{j}$ are disjoint to control the second term. Hence, it remains to understand under what conditions one has

$$
\limsup _{\varepsilon \rightarrow 0} \mathbb{E} \frac{\varepsilon^{6}}{\lambda_{0}^{2}} \sum_{i} \int_{B_{i}}\left|\sum_{j \neq i} \nabla V\left(x-x_{j}\right) \nabla \varphi\left(x_{j}\right)\right|^{2} d x \rightarrow 0, \quad \lambda \rightarrow 0 .
$$


Let $\tilde{B}_{i}=B\left(x_{i}, c_{0}\left(\lambda / \lambda_{0}\right)^{1 / 3} \varepsilon\right)$, with $c_{0}=\left(\frac{3}{4 \pi}\right)^{1 / 3}$, so that almost surely $r_{n} \sim c_{0}\left(\lambda / \lambda_{0}\right)^{1 / 3} \varepsilon$ as $\varepsilon \rightarrow 0$. Introducing

$$
\tilde{I}_{\varepsilon}=\frac{\varepsilon^{6}}{\lambda_{0}^{2}} \sum_{i} \int_{\tilde{B}_{i}}\left|\sum_{j \neq i} \nabla V\left(x-x_{j}\right) \nabla \varphi\left(x_{j}\right)\right|^{2} d x
$$

we show as in the case of $I_{\varepsilon}$ that it is bounded uniformly in $\varepsilon$ and $\omega$, and moreover that

$$
\begin{aligned}
\left|I_{\varepsilon}-\tilde{I}_{\varepsilon}\right| & \leq C_{\lambda} \sum_{i} \int_{B_{i} \Delta \tilde{B}_{i}}\left|\nabla^{2} \Delta^{-1} 1_{B_{i}} \nabla \varphi\left(x_{i}\right)\right|^{2}+C_{\lambda} \int_{\cup\left(B_{i} \Delta \tilde{B}_{i}\right)}\left|\nabla^{2} \Delta^{-1} \sum_{j} 1_{B_{j}} \nabla \varphi\left(x_{j}\right)\right|^{2} \\
& \leq C_{\lambda}^{\prime}\left|1-\frac{c_{0} \lambda^{1 / 3} \varepsilon}{\lambda_{0}^{1 / 3} r_{n}}\right|+C_{\lambda}\left\|\sum_{i} 1_{B_{i} \Delta \tilde{B}_{i}}\right\| L_{L^{2}}\left\|\sum_{j} 1_{B_{j}} \nabla \varphi\left(x_{j}\right)\right\|_{L^{4}}^{2} \\
& \leq C_{\lambda}^{\prime \prime}\left(\left|1-\frac{c_{0} \lambda^{1 / 3} \varepsilon}{\lambda_{0}^{1 / 3} r_{n}}\right|+\left|1-\frac{c_{0} \lambda^{1 / 3} \varepsilon}{\lambda_{0}^{1 / 3} r_{n}}\right|^{1 / 2}\right) \rightarrow 0, \quad \text { as } \varepsilon \rightarrow 0 .
\end{aligned}
$$

We used the continuity of $\nabla^{2} \Delta^{-1}$ over $L^{4}$ in the second inequality. By the dominated convergence theorem, the final step is to understand when

$$
\limsup _{\varepsilon \rightarrow 0} \mathbb{E} \frac{\varepsilon^{6}}{\lambda_{0}^{2}} \sum_{i} \int_{\tilde{B}_{i}}\left|\sum_{j \neq i} \nabla V\left(x-x_{j}\right) \nabla \varphi\left(x_{j}\right)\right|^{2} d x \rightarrow 0, \quad \lambda \rightarrow 0 .
$$

The advantage of $\tilde{B}_{i}$ over $B_{i}$ is that its radius is not random anymore. We write

$$
\begin{aligned}
& \mathbb{E} \frac{\varepsilon^{6}}{\lambda_{0}^{2}} \sum_{i} \int_{\tilde{B}_{i}}\left|\sum_{j \neq i} \nabla V\left(x-x_{j}\right) \nabla \varphi\left(x_{j}\right)\right|^{2} d x=\mathbb{E} \frac{\varepsilon^{3}}{\lambda_{0}^{2}} \sum_{i} \int_{\varepsilon^{-1} \tilde{B}_{i}}\left|\sum_{j \neq i} \nabla V\left(y-y_{j}\right) \nabla \varphi\left(x_{j}\right)\right|^{2} d y \\
= & \mathbb{E} \frac{\varepsilon^{3}}{\lambda_{0}^{2}} \sum_{i \neq j} \int_{\varepsilon^{-1} \tilde{B}_{i}}\left|\nabla V\left(y-y_{j}\right) \nabla \varphi\left(x_{j}\right)\right|^{2} d y \\
+ & \mathbb{E} \frac{\varepsilon^{3}}{\lambda_{0}^{2}} \sum_{i \neq j \neq j^{\prime}} \int_{\varepsilon^{-1} \tilde{B}_{i}} \nabla V\left(y-y_{j}\right) \nabla \varphi\left(x_{j}\right) \cdot \nabla V\left(y-y_{j^{\prime}}\right) \nabla \varphi\left(x_{j^{\prime}}\right) d y .
\end{aligned}
$$

Using the definition of the $k$-point correlation functions $\rho_{k}$, see [4, p18], we find

$$
\begin{aligned}
& \mathbb{E} \frac{\varepsilon^{3}}{\lambda_{0}^{2}} \sum_{i \neq j} \int_{\varepsilon^{-1} \tilde{B}_{i}}\left|\nabla V\left(y-y_{j}\right) \nabla \varphi\left(x_{j}\right)\right|^{2} d y \\
= & \frac{\varepsilon^{3}}{\lambda_{0}^{2}} \int_{\left(\varepsilon^{-1} \mathcal{O}\right)^{2}} d z d z^{\prime} \rho_{2}\left(z, z^{\prime}\right) \int_{B\left(z, c_{0}\left(\lambda / \lambda_{0}\right)^{1 / 3}\right)}\left|\nabla V\left(y-z^{\prime}\right) \nabla \varphi\left(\varepsilon z^{\prime}\right)\right|^{2} d y \\
\leq & C \frac{\varepsilon^{3}}{\lambda_{0}^{2}} \int_{\left(\varepsilon^{-1} \mathcal{O}\right)^{2}} d z d z^{\prime} \rho_{2}\left(z, z^{\prime}\right) \int_{B\left(z, c_{0}\left(\lambda / \lambda_{0}\right)^{1 / 3}\right)} \frac{1}{\left|y-z^{\prime}\right|^{6}} d y
\end{aligned}
$$

By stationarity, $\rho_{2}\left(z, z^{\prime}\right)=\rho_{2}\left(0, z^{\prime}-z\right)$. After a change of variable, we get

$$
\mathbb{E} \frac{\varepsilon^{3}}{\lambda_{0}^{2}} \sum_{i \neq j} \int_{\tilde{B}_{i}}\left|\nabla V\left(y-y_{j}\right) \nabla \varphi\left(x_{j}\right)\right|^{2} d y \leq C \frac{|\mathcal{O}|}{\lambda_{0}^{2}} \int_{\mathbb{R}^{3}} \int_{B\left(0, c_{0}\left(\lambda / \lambda_{0}\right)^{1 / 3}\right)} \frac{\rho_{2}\left(0, z^{\prime}\right)}{\left|y-z^{\prime}\right|^{6}} d y d z^{\prime} .
$$

Under assumption (35) we find eventually that the r.h.s. goes to zero if and only if

$$
\frac{\lambda}{\lambda_{0}^{3}} \int_{\mathbb{R}^{3}} \frac{\rho_{2}\left(0, z^{\prime}\right)}{\left|z^{\prime}\right|^{6}+\left(\lambda / \lambda_{0}\right)^{2}} d z^{\prime} \rightarrow 0
$$


As regards the other term, we get

$$
\begin{aligned}
& \mathbb{E} \frac{\varepsilon^{3}}{\lambda_{0}^{2}} \sum_{i \neq j \neq j^{\prime}} \int_{\varepsilon^{-1} \tilde{B}_{i}} \nabla V\left(y-y_{j}\right) \nabla \varphi\left(x_{j}\right) \cdot \nabla V\left(y-y_{j^{\prime}}\right) \nabla \varphi\left(x_{j^{\prime}}\right) d y \\
= & \mathbb{E} \frac{\varepsilon^{3}}{\lambda_{0}^{2}} \int_{\left(\varepsilon^{-1} \mathcal{O}\right)^{3}} d z d z^{\prime} d z^{\prime \prime} \rho_{3}\left(z, z^{\prime}, z^{\prime \prime}\right) \int_{B\left(z, c_{0}\left(\lambda / \lambda_{0}\right)^{1 / 3}\right)} \nabla V\left(y-z^{\prime}\right) \nabla \varphi\left(\varepsilon z^{\prime}\right) \cdot \nabla V\left(y-z^{\prime \prime}\right) \nabla \varphi\left(\varepsilon z^{\prime \prime}\right) d y \\
= & \mathbb{E} \frac{\varepsilon^{3}}{\lambda_{0}^{2}} \int_{\left(\varepsilon^{-1} \mathcal{O}\right)^{3}} d z d z^{\prime} d z^{\prime \prime} \rho_{3}\left(0, z^{\prime}, z^{\prime \prime}\right) \int_{B\left(0, c_{0}\left(\lambda / \lambda_{0}\right)^{1 / 3}\right)} \nabla V\left(y-z^{\prime}\right) \nabla \varphi\left(\varepsilon\left(z+z^{\prime}\right)\right) \cdot \nabla V\left(y-z^{\prime \prime}\right) \nabla \varphi\left(\varepsilon\left(z+z^{\prime \prime}\right)\right) d y
\end{aligned}
$$

where we have used stationarity: $\rho_{3}\left(z, z^{\prime}, z^{\prime \prime}\right)=\rho_{3}\left(0, z^{\prime}-z, z^{\prime \prime}-z\right)$ and a change of variable to obtain the last line. Finally,

$$
\begin{aligned}
& \left|\mathbb{E} \frac{\varepsilon^{3}}{\lambda_{0}^{2}} \sum_{i \neq j \neq j^{\prime}} \int_{\varepsilon^{-1} \tilde{B}_{i}} \nabla V\left(y-y_{j}\right) \nabla \varphi\left(x_{j}\right) \cdot \nabla V\left(y-y_{j^{\prime}}\right) \nabla \varphi\left(x_{j^{\prime}}\right) d y\right| \\
\leq & |\mathcal{O}| \sup _{x \in \mathbb{R}^{3}} \int_{B\left(0, c_{0}\left(\lambda / \lambda_{0}\right)^{1 / 3}\right)}\left(\frac{\partial}{\partial z_{i}^{\prime}} \frac{\partial}{\partial z_{k}^{\prime}} \Delta_{z^{\prime}}^{-1}\right)\left(\frac{\partial}{\partial z_{i}^{\prime \prime}} \frac{\partial}{\partial z_{j}^{\prime \prime}} \Delta_{z^{\prime \prime}}^{-1}\right) A_{x, k, j}^{\varepsilon}\left(z^{\prime}=y, z^{\prime \prime}=y\right) d y
\end{aligned}
$$

where $A_{x, k, j}^{\varepsilon}\left(z^{\prime}, z^{\prime \prime}\right)=1_{\varepsilon^{-1} \mathcal{O}}\left(z^{\prime}\right) 1_{\varepsilon^{-1} \mathcal{O}}\left(z^{\prime \prime}\right) \rho_{3}\left(0, z^{\prime}, z^{\prime \prime}\right) \partial_{k} \varphi\left(x+\varepsilon z^{\prime}\right) \partial_{j} \varphi\left(x+\varepsilon z^{\prime \prime}\right)$.

Although this last quantity is a bit intricate, we may expect that it goes to zero with $\lambda$ under reasonable assumptions on the process. For instance, if the process is Poisson of intensity $\lambda_{0}$ (neglecting previous assumptions on the minimal distance), then $\rho_{3}=\lambda_{0}^{3}$, and $\left|A_{x, k, j}^{\varepsilon}\left(z^{\prime}, z^{\prime \prime}\right)\right| \leq C \lambda_{0}^{3}$, resulting in

$$
\left|\mathbb{E} \frac{\varepsilon^{3}}{\lambda_{0}^{2}} \sum_{i \neq j \neq j^{\prime}} \int_{\varepsilon^{-1} \tilde{B}_{i}} \nabla V\left(y-y_{j}\right) \nabla \varphi\left(x_{j}\right) \cdot \nabla V\left(y-y_{j^{\prime}}\right) \nabla \varphi\left(x_{j^{\prime}}\right) d y\right|=O(\lambda) .
$$

The condition (37) is more stringent: for the Poisson process of intensity $\lambda_{0}$, where $\rho_{2}=\lambda_{0}^{2}$, we find that the quantity at the left-hand side of (37) is $O(1)$ as $\lambda \rightarrow 0$, but non-vanishing. Hence, our result does not cover this case: to cover it, we would need a weaker criterion than (A2), in the same way as the criterion (12) derived in [34] is weaker than (H2) or (H2 ${ }^{\sharp}$. Still, (37) is fulfilled by much more configurations than those satisfying (11) almost surely. Indeed, this latter case corresponds to $\rho_{2}\left(0, z^{\prime}\right)=0$ for $\left|z^{\prime}\right| \geq c \lambda_{0}^{-1 / 3}$, so that the quantity at the left-hand side of $(37)$ is $O(\lambda)$, much stronger than the $o(1)$ asked in $\left(\mathrm{A} 2_{b}\right)$.

\section{Acknowledgements}

The author acknowledges the support of the Institut Universitaire de France, and of the SingFlows project, grant ANR-18-CE40-0027 of the French National Research Agency (ANR).

\section{References}

[1] G. Allaire. Homogenization of the Navier-Stokes equations in open sets perforated with tiny holes. I. Abstract framework, a volume distribution of holes. Arch. Rational Mech. Anal., 113(3):209-259, 1990.

[2] H. Ammari, P. Garapon, H. Kang, and H. Lee. Effective viscosity properties of dilute suspensions of arbitrarily shaped particles. Asymptot. Anal., 80(3-4):189-211, 2012.

[3] H. Ammari and H. Zhang. Effective medium theory for acoustic waves in bubbly fluids near Minnaert resonant frequency. SIAM J. Math. Anal., 49(4):3252-3276, 2017.

[4] A. Borodin and S. Serfaty. Renormalized energy concentration in random matrices. Comm. Math. Phys., 320(1):199-244, 2013. 
[5] H. C. Brinkman. A calculation of the viscous force exerted by a flowing fluid on a dense swarm of particles. Flow, Turbulence and Combustion, 1(1):27, Dec 1949.

[6] L. A. Caffarelli and A. Mellet. Random homogenization of an obstacle problem. Ann. Inst. H. Poincaré Anal. Non Linéaire, 26(2):375-395, 2009.

[7] C. Calvo-Jurado, J. Casado-Díaz, and M. Luna-Laynez. Homogenization of nonlinear Dirichlet problems in random perforated domains. Nonlinear Anal., 133:250-274, 2016.

[8] D. Cioranescu and F. Murat. A strange term coming from nowhere. In Topics in the mathematical modelling of composite materials, volume 31 of Progr. Nonlinear Differential Equations Appl., pages 4593. Birkhäuser Boston, Boston, MA, 1997.

[9] R. Clausius. Die mechanische Behandlung der Elektricität. Vieweg, Braunshweig, 1879.

[10] G. Dal Maso. Asymptotic behaviour of minimum problems with bilateral obstacles. Ann. Mat. Pura Appl. (4), 129:327-366 (1982), 1981.

[11] G. Dal Maso and P. Longo. Г-limits of obstacles. Ann. Mat. Pura Appl. (4), 128:1-50, 1981.

[12] D. J. Daley and D. Vere-Jones. An introduction to the theory of point processes. Vol. I. Probability and its Applications (New York). Springer-Verlag, New York, second edition, 2003. Elementary theory and methods.

[13] D. J. Daley and D. Vere-Jones. An introduction to the theory of point processes. Vol. II. Probability and its Applications (New York). Springer, New York, second edition, 2008. General theory and structure.

[14] L. Desvillettes, F. Golse, and V. Ricci. The mean-field limit for solid particles in a Navier-Stokes flow. J. Stat. Phys., 131(5):941-967, 2008.

[15] M. Duerinckx and A. Gloria. Analyticity of homogenized coefficients under Bernoulli perturbations and the Clausius-Mossotti formulas. Arch. Ration. Mech. Anal., 220(1):297-361, 2016.

[16] A. Einstein. Eine neue bestimmung der moleküldimensionen. Ann. Physik., 19:289-306, 1906.

[17] G. P. Galdi. An introduction to the mathematical theory of the Navier-Stokes equations. Vol. I, volume 38 of Springer Tracts in Natural Philosophy. Springer-Verlag, New York, 1994. Linearized steady problems.

[18] D. Gérard-Varet and M. Hillairet. Analysis of the viscosity of dilute suspensions beyond Einstein's formula. arXiv:1905.08208, May 2019.

[19] A. Giunti and R. Höfer. Homogenization for the Stokes equations in randomly perforated domains under almost minimal assumptions on the size of the holes. arXiv:1809.04491, September 2018.

[20] A. Giunti, R. Höfer, and J. J. L. Velázquez. Homogenization for the Poisson equation in randomly perforated domains under minimal assumptions on the size of the holes. Comm. Partial Differential Equations, 43(9):1377-1412, 2018.

[21] A. Gloria. A scalar version of the Caflisch-Luke paradox. arXiv:1907.08182, July 2019.

[22] B. M. Haines and A. L. Mazzucato. A proof of Einstein's effective viscosity for a dilute suspension of spheres. SIAM J. Math. Anal., 44(3):2120-2145, 2012.

[23] M. Hillairet. On the homogenization of the Stokes problem in a perforated domain. Arch. Ration. Mech. Anal., 230(3):1179-1228, 2018.

[24] M. Hillairet and D. Wu. Effective viscosity of a polydispersed suspension. Preprint arXiv:1905.12306, 2019.

[25] R. M. Höfer. The inertialess limit of particle sedimentation modeled by the Vlasov-Stokes equations. SIAM J. Math. Anal., 50(5):5446-5476, 2018.

[26] P.-E. Jabin and F. Otto. Identification of the dilute regime in particle sedimentation. Comm. Math. Phys., 250(2):415-432, 2004.

[27] T. Lévy and E. Sánchez-Palencia. Einstein-like approximation for homogenization with small concentration. II. Navier-Stokes equation. Nonlinear Anal., 9(11):1255-1268, 1985.

[28] V. A. Marčenko and E. J. Hruslov. Estimation of the accuracy of approximation of solutions of boundary value problems with a fine-grained boundary. In Problems in mechanics and mathematical physics (Russian), pages 208-223, 298. 1976.

[29] J. Maxwell Garnett. Colours in metal glasses and in metallic films. Philosophical Transactions of the Royal Society of London. Series A, 203, 1904.

[30] A. Mecherbet. Sedimentation of particles in Stokes flow. arXiv:1806.07795, June 2018.

[31] O. Mossotti. Discussione analitica sul'influenza che l'azione di un mezzo dielettrico ha sulla distribuzione dell'elettricità alla superficie di più corpi elettrici disseminati in esso. Mem. Mat. Fis. della Soc. Ital. di Sci. in Modena, 24:49-74, 1850. 
[32] B. Niethammer and R. Schubert. A local version of Einstein's formula for the effective viscosity of suspensions. arXiv:1903.08554.

[33] S. Ozawa. Point interaction potential approximation for $(-\Delta+U)^{-1}$ and eigenvalues of the Laplacian on wildly perturbed domain. Osaka J. Math., 20(4):923-937, 1983.

[34] G. C. Papanicolaou and S. R. S. Varadhan. Diffusion in regions with many small holes. In Stochastic differential systems (Proc. IFIP-WG 7/1 Working Conf., Vilnius, 1978), volume 25 of Lecture Notes in Control and Information Sci., pages 190-206. Springer, Berlin-New York, 1980.

[35] E. Sánchez-Palencia. Einstein-like approximation for homogenization with small concentration. I. Elliptic problems. Nonlinear Anal., 9(11):1243-1254, 1985. 